\title{
ON PRIMITIVELY 2-UNIVERSAL QUADRATIC FORMS
}

\author{
N. V. BUDARINA
}

\begin{abstract}
The primitive representations of binary positive definite, classically integral quadratic forms over the local rings $\mathbb{Z}_{p}$ are studied. For the global ring, an efficient method is obtained for determining when a quadratic form is primitively 2-universal.
\end{abstract}

\section{$\S 1$. INTRODUCTION}

In 1993, J. Conway and V. Schneeberger announced the 15-theorem, in which they gave a criterion for determining when a positive definite, classically integral (or with an integer matrix) quadratic form represents all positive integers. More precisely, they proved (unpublished) that any positive definite, classically integral quadratic form that represents the set of nine critical numbers

$$
S_{1}=\{1,2,3,5,6,7,10,14,15\}
$$

is universal. In 1999, M. Bhargava published the paper [1, in which a simpler proof of the 15 -theorem was given. He also showed that there exist exactly 204 universal positive definite, classically integral quaternary quadratic forms.

Today we can indicate at least four directions in which the universality problem for quadratic forms is being developed:

- the multidimensional analog of universal forms ( $m$-universal forms) (see 2, 3]);

- universal forms over (totally) real number fields (see [4, 8]);

- almost universal forms (see [9]-[13]);

- "even" / "odd" / "prime" universality (representation of all even/odd/ prime numbers) (see [14, 15]).

Then, the problem of studying primitively universal forms arises naturally. Primitive representations play an important role in finding the number of representations. A quadratic form $Q$ is said to be (primitively) m-universal if it represents (primitively) all positive definite, integral forms $A$ of dimension $m$. The universal forms are closely related to the so-called regular forms. A positive definite, integral quadratic form $Q$ is said to be $m$-regular if $Q$ represents each quadratic form of dimension $m$ represented by the genus $[Q]$. It is obvious that every $m$-universal form is an $m$-regular form. In [16], Oh proved that for $m \geq 27$, every $m$-regular form is $m$-universal.

In [17, Earnest proved that there exist only finitely many 2-regular primitive, positive definite, integral quaternary quadratic forms. Later, B. Kim, M. Kim, and Oh [3] showed that 2-universal quaternary quadratic forms do not exist. (Consequently, $\operatorname{dim} Q=5$ is the smallest possible dimension for the 2-universal integral quadratic forms $Q$.) They found all positive definite, classically integral quadratic forms of dimension 5 that represent all positive definite, classically integral, binary quadratic forms (see Table 9), and they

2010 Mathematics Subject Classification. Primary 11E08.

Key words and phrases. Primitively universal quadratic forms, $p$-adic symbols.

Supported by RFBR (grants nos. 08-01-00326 and 07-01-00306). 
obtained a similar criterion for 2-universality (see [3]):

$$
S_{2}=\left\{\left(\begin{array}{ll}
1 & 0 \\
0 & 1
\end{array}\right),\left(\begin{array}{ll}
2 & 0 \\
0 & 3
\end{array}\right),\left(\begin{array}{ll}
3 & 0 \\
0 & 3
\end{array}\right),\left(\begin{array}{ll}
2 & 1 \\
1 & 2
\end{array}\right),\left(\begin{array}{ll}
2 & 1 \\
1 & 3
\end{array}\right),\left(\begin{array}{ll}
2 & 1 \\
1 & 4
\end{array}\right)\right\} .
$$

We consider primitive representations of quadratic forms; our studies are devoted to searching for similar efficient criteria of universality for primitive representations. In our paper [18, we presented an efficient method for determining when a positive definite, classically integral quadratic form of dimension at least four and with square-free odd determinant is primitively almost universal. A quadratic form is said to be (primitively) almost universal if it represents (primitively) all positive integers except for finitely many of them.

Theorem 1 (see [18). Let $Q$ be a positive definite, classically integral quadratic form of dimension at least 4 with square-free odd determinant. Then the form $Q$ is primitively almost universal if $Q$ primitively represents over $\mathbb{Z}$ each positive integer in the set $S_{3}=$ $\{1,4,8\}$. If the form $Q$ with the properties described above is unique in its genus $[Q]$ (i.e., $h(Q)=1$ ), then $Q$ is a primitively universal form.

In the present paper, we study primitive representations of binary forms over the local rings $\mathbb{Z}_{p}$. An integral form $Q$ is said to be locally primitively 2 -universal if $Q$ is primitively $p \sim$ universal for every prime $p$, i.e., if $Q$ represents all binary forms with integral $p$-adic coefficients over the ring $\mathbb{Z}_{p}$. In $\S 3$, devoted to primitive representations of forms over an odd local ring $\mathbb{Z}_{p}$, we obtain a result on primitive $p \sim$ universality of some special forms $Q$. $\S 4$ is important and new; in it we construct minimal embeddings of forms $A$ in forms $Q$ of odd determinants over the ring $\mathbb{Z}_{2} . \oint 5$ contains the main results and their applications. In particular, we obtain an efficient method for determining when a quadratic form is primitively 2-universal.

Theorem 2. Let $Q$ be a positive definite, classically integral quadratic form of dimension at least 5 with square-free odd determinant $|Q|$, and let $h(Q)=1$. Then the form $Q$ is primitively 2-universal if for every odd prime $p$ dividing $|Q|$, the form $Q$ primitively represents over $\mathbb{Z}$ each binary form in the set

$$
S_{4, p}=\left\{\left(\begin{array}{ll}
1 & 0 \\
0 & 1
\end{array}\right), 2\left(\begin{array}{ll}
2 & 1 \\
1 & 2
\end{array}\right), 8\left(\begin{array}{ll}
1 & 0 \\
0 & 7
\end{array}\right), p^{2}\left(\begin{array}{ll}
1 & 0 \\
0 & 1
\end{array}\right)\right\} .
$$

Example 1. Consider the quadratic forms $Q_{1}=3 x_{1}^{2}+\sum_{j=2}^{6} x_{j}^{2}$ and $Q_{k}=\sum_{i=1}^{k} x_{i}^{2}$, $5 \leq k \leq 8$, which are one-class forms (i.e., $h\left(Q_{i}\right)=1$ ). The forms $Q_{6}, Q_{7}$, and $Q_{8}$ are primitively 2-universal, and the forms $Q_{1}, Q_{5}$ are not.

\section{$\S 2$. Primitive Representations of Forms}

We pass from the ring $\mathbb{Z}$ to the ring of $p$-adic integers $\mathbb{Z}_{p}$ and consider primitive representations over the local ring $\mathbb{Z}_{p}$. We denote by $\mathbb{Z}_{p}^{\times}$the group of units of the $\operatorname{ring} \mathbb{Z}_{p}$.

2.1. Let $Q={ }^{t} Q$ be a nonsingular symmetric matrix of size $n$ in the set $M_{n}\left(\mathbb{Z}_{p}\right)$ of matrices with entries in $\mathbb{Z}_{p}$, and let $A={ }^{t} A$ be a similar matrix in $M_{m}\left(\mathbb{Z}_{p}\right)$ of dimension $1 \leq m<n$. We identify the matrices $Q$ and $A$ with the quadratic forms determined by them. The form $A$ is primitively represented by the form $Q$ if there exists a matrix $b \in M_{n, m}\left(\mathbb{Z}_{p}\right)$ satisfying

$$
Q[b]={ }^{t} b Q b=A .
$$

Moreover, $b$ is primitive, i.e., the greatest common divisor $d(b)$ of the minors of size $m$ of $b$ is equal to the unity of the ring $\mathbb{Z}_{p}$. The primitivity of $b$ is equivalent to the existence of 
an augmented matrix $B=(b \mid e)$ belonging to the unimodular group $S L_{n}^{ \pm}\left(\mathbb{Z}_{p}\right)$ of matrices $M$ with entries in $\mathbb{Z}_{p}$ and with determinant $|M|= \pm 1$. With respect to the group $S L_{n}^{ \pm 1}\left(\mathbb{Z}_{p}\right)$, the form $Q$ is equivalent to the integral form

$$
Q_{B}=Q[B]=\left(\begin{array}{cc}
A & C \\
{ }^{t} C & Q^{\prime}
\end{array}\right) .
$$

The matrix $M_{C}=\left(\begin{array}{cc}1_{m} & -A^{-1} C \\ 0 & 1_{n-m}\end{array}\right)$ belonging to $S L_{n}^{ \pm}\left(\mathbb{Q}_{p}\right)$ takes $Q_{B}$ to the form

$$
Q_{B}\left[M_{C}\right]=\left(\begin{array}{cc}
A & 0 \\
0 & G^{\prime}
\end{array}\right)=A \oplus G^{\prime}
$$

which is equal to the direct sum of the forms $A$ and $G^{\prime}=Q^{\prime}-A^{-1}[C]$. We fix a nonzero $p$-integer $a=\{a\} \cdot|a|_{p}^{-1}$, where $|a|_{p}=p^{-\nu_{p}(a)}$ denotes the $p$-norm of $a$, such that the matrix $a A^{-1}$ has integral entries and $\nu_{p}(a)=0,1,2, \ldots$ is minimal under this condition, i.e., $a$ is the $p$-adic analog of the level of a quadratic form over the ring $\mathbb{Z}$ of rational integers. Then we obtain the decomposition

$$
a Q_{B}\left[M_{C}\right]=\left(\begin{array}{cc}
a A & 0 \\
0 & G
\end{array}\right)
$$

where $G=G_{Q, A}=a Q^{\prime}-E[C]$ is a $p$-integer symmetric nonsingular matrix of size $k=n-m>0$ and $E=a A^{-1}$ is an integral matrix. The block $Q^{\prime}$ is integral; therefore, $C$ is contained in the set $c_{G}^{A}$ of solutions of the matrix congruence

$$
E[C] \equiv-G \quad\left(\bmod a \mathbb{Z}_{p}\right), \quad C \in M_{m, k}\left(\mathbb{Z}_{p}\right) .
$$

From (44) we deduce that the determinant

$$
|G|=\frac{a^{n-m}|Q|}{|A|}
$$

of the form $G$ does not depend on the choice of the primitive representation $b$ in (10).

If we exclude the dependence on the choice of $B$ from the sequence $b \rightarrow B \rightarrow C \rightarrow G$, we obtain a map

$$
[Q, A]: b \mapsto\{G\}=\{G(b)\},
$$

where $b$ determines the class $\{G(b)\}$ rather than the form $G(b)$ itself (see [19]). The equivalence classes $\{G\}$ of forms are considered relative to $S L^{ \pm}\left(\mathbb{Z}_{p}\right)$. We denote by $p b_{Q, A}$ the set of all primitive representations $b$ in (10) and by $p b_{Q, A}(\{G\})$ the set of representations $b$ corresponding to the class $\{G\}$. Since $|G|$ is determined by the forms $A$ and $Q$, it follows that $p b_{Q, A}$ splits into a finite number of sets $p b_{Q, A}(\{G\})$ :

$$
p b_{Q, A}=\sum_{\{G\}} p b_{Q, A}(\{G\}) .
$$

Conversely, for an arbitrary solution $G$ as in (5), the form

$$
Q_{G}^{A}(C)=\frac{1}{a}\left(\begin{array}{cc}
a A & 0 \\
0 & G
\end{array}\right)\left[M_{C}^{-1}\right]=\left(\begin{array}{cc}
A & C \\
{ }^{t} C & \frac{1}{a}(E[C]+G)
\end{array}\right)
$$

is integral and is equivalent to the initial form $Q$.

Let $C_{G}^{A}(Q)$ be the set of matrices $C$ for which the form (8) belongs to the class $\{Q\}$. Now we fix a class $\{G\}$ and exclude the dependence on $B$ from $b \rightarrow B \rightarrow C$, obtaining orbits $\{C\}=\left\{C V / \bmod A: V \in O_{\mathbb{Z}_{p}}(G)\right\}$ of solutions $C$ of (5). The map $b \rightarrow\{C\}$ splits the solutions $b$ of (1) into orbits $\{b\}=\left\{\gamma b: \gamma \in O_{\mathbb{Z}_{p}}(Q)\right\}$ relative to the group $O(Q)$ over $\mathbb{Z}_{p}$. The quotient map

$$
p b_{Q, A}(\{G\}) \supset\{b\} \rightrightarrows\{C\} \subset C_{G}^{A}(Q)
$$

is a bijection (see [19]). 
Two forms $A$ and $G$ are coupled if the set $\operatorname{Ext}(G, A)$ of coupling matrices $C$ from (8) is nonempty. The partition (7) has a dual partition

$$
\operatorname{Ext}(G, A)=\sum_{Q} C_{G}^{A}(Q)
$$

which is finite by (6).

The case of coprime $a$ and $|Q|$ ( $a$ is the level of the form $A$ ) was studied in [19], where it was shown, for example, that for odd $p$ 's that do not divide $|Q|$, the class $\{G\}_{\mathbb{Z}_{p}}$ is uniquely determined by the forms $A$ and $Q$. In the case where $a$ and $|Q|$ have a common divisor, the representations (11) ramify, and the form $A$ and the genus $[Q]$ no longer determine the genus $[G]$ of forms $G$ uniquely. In particular, this is shown by the partition into different orbits $\{b\}$ of the set of primitive representations $b: Q[b]=A$ over the local rings $\mathbb{Z}_{p}$ for primes $p$ that divide both $a$ and $|Q|$. All the orbits are split into the sets $p b_{Q, A}(\{G\})$ enumerated by a finite number of classes $\{G\}=\{G\}_{\mathbb{Z}_{p}}$, and this yields a "rough" classification of orbits. Inside the above sets, there is a one-to-one correspondence between the orbits $\{b\}$ and the $O(G)$-orbits $\{C\}$ of matrices $C$ that couple the forms $A$ and $Q$; this is a "subtle" classification of the orbits $\{b\}$. The ramification of representations over $\mathbb{Z}_{p}, p \neq 2$, along with arithmetic applications, were presented in [20, 21, 22]. Difficulties emerge in considering the representations of forms over the even ring $\mathbb{Z}_{2}$, which ramify even if the determinants are relatively prime.

2.2. At the beginning of the paper, we used the language of quadratic forms, but sometimes it is more convenient to use the language of lattices. By a lattice $L$ we mean a free $\mathbb{Z}_{p}$-module of finite rank with nondegenerate symmetric bilinear form $(\cdot, \cdot)_{L}$ with values in the ring $\mathbb{Z}_{p}$. If $e=\left(e_{1}, \ldots, e_{n}\right)$ is a basis of the lattice $L$, then $Q={ }^{t} e \cdot e=\left(\left(e_{i}, e_{j}\right)_{L}\right)_{n \times n}$ is its Gram matrix, which is integral and nonsingular. If $e_{0}=e U$ is another basis of $L$, then $U$ is an integral matrix with determinant in $\mathbb{Z}_{p}^{\times}$, and the Gram matrix $Q_{0}={ }^{t} e_{0} \cdot e_{0}=Q[U]$ is equivalent to $Q$. Identifying isomorphic lattices, we obtain a bijection $L \geq\{Q\}$.

An embedding $i: M \hookrightarrow L$ of a lattice $M$ of dimension $1 \leq m \leq n$ in $L$ is an embedding of the $\mathbb{Z}_{p}$-module $M$ in $L$ satisfying $\left(x^{\prime}, y^{\prime}\right)_{M}=\left(i\left(x^{\prime}\right), i\left(y^{\prime}\right)\right)_{L}$. Let $e^{\prime}$ be a basis of the lattice $M$, and let $A={ }^{t} e^{\prime} \cdot e^{\prime}$ be the Gram matrix. Then $i\left(x^{\prime}\right)=b \cdot x^{\prime}$ for $b \in M_{n, m}\left(\mathbb{Z}_{p}\right)$ and, moreover, $Q[b]=A$. We shall write $b: A \hookrightarrow Q$ and say that the quadratic form $A$ is embedded in the form $Q$. Conversely, any embedding $b$ of the form $A$ in $Q$ gives an embedding $i$ of the lattice $M$ in $L$. Thus, embeddings of lattices give rise to embeddings of quadratic forms for the bases chosen in the lattices.

For lattices, the primitivity of an embedding $i: M \hookrightarrow L$ means that $L / i(M)$ is a free $\mathbb{Z}_{p}$-module. Next, an embedding $i$ is decomposable, i.e., $i=i_{1} \oplus i_{2}$, if there exist decompositions of lattices into the orthogonal sums $M=M_{1} \oplus M_{2}, \operatorname{dim} M_{j} \geq 1$, $j=1,2$, and $L=L_{1} \oplus L_{2}$ such that the restrictions $i_{j}=\left.i\right|_{M_{j}}$ determine embeddings $i_{j}: M_{j} \hookrightarrow L_{j}, j=1,2$. If the inclusion $i M \subset L_{1}$ into an arbitrary sublattice $L_{1}$ and the decomposition $L=L_{1} \oplus L_{2}$ imply that $L_{1}=L$, then $i$ is called the minimal embedding of dimension $\operatorname{dim} L$.

\section{§3. Primitive Representations of Forms over An OdD LOCAL Ring}

Consider local representations of forms $A$ by forms $Q$ over $\mathbb{Z}_{p}$. Moreover, it is necessary to distinguish the case of odd $p$ from the case where $p=2$.

For $p$ odd, for a nondegenerate form $Q \in M_{n}\left(\mathbb{Z}_{p}\right)$ the $p$-adic symbol can be represented (see [23]) as

$$
p_{Q}=\prod_{q} q^{\epsilon_{q}(Q) n_{q}(Q)}, \quad q=p^{\alpha}, \quad \alpha=0,1,2, \ldots
$$


This means that the matrix $Q$ over $\mathbb{Z}_{p}$ has the Jordan decomposition $Q=\sum_{q} q Q_{q}$, where the forms $Q_{q} \in G L\left(\mathbb{Z}_{p}\right)$ have dimensions $n_{q}(Q)$ and signs $\epsilon_{q}(Q)=\left(\frac{\left|Q_{q}\right|}{p}\right) ;\left(\frac{*}{p}\right)$ is the Legendre symbol. The summands $q Q_{q}$ are called the Jordan constituents of scale $q$. Existence conditions for the Jordan constituents $q Q_{q}$ can be found in [23].

3.1. First, we consider quadratic forms with $\mathbb{Z}_{p}$-integral symmetric matrices $Q=Q_{1}$ of determinant $\left|Q_{1}\right| \not \equiv 0(\bmod p)$ for odd prime $p$. Using the reduction theory for quadratic forms over the local ring $\mathbb{Z}_{p}, p \neq 2$ (see [24]), we may assume that $A=A_{1} \oplus A_{\geq p}$, where the block $A_{1}$ has maximal dimension under the condition $\left|A_{1}\right| \not \equiv 0(\bmod p)$. Denote by $a=p^{\alpha}$ the level of the form $A$ over $\mathbb{Z}_{p}$. If the odd prime $p$ divides $\left|Q_{1}\right|$, then the integral form $G$ in the congruence (5) must satisfy the following restriction over $\mathbb{Z}_{p}$ (see [19, (6.14)]):

$$
G \equiv\left(\begin{array}{cc}
-a A_{\geq p}^{-1} & 0 \\
0 & 0
\end{array}\right) \quad(\bmod a) .
$$

For this reason, we assume that

$$
G=G_{<a} \oplus a G_{a} \text { where } G_{<a}=-a A_{\geq p}^{-1} .
$$

The set $C_{G}^{A}=c_{G}^{A} / \bmod A$ contains a unique orbit $\{C\}$ composed of the classes $C S / \bmod$ $A$, where $S$ ranges over the group of integral automorphisms $O_{\mathbb{Z}_{p}}(G)$. Using the bijection (9) between the orbits $\{C\}$ and the $O_{\mathbb{Z}_{p}}(Q)$-orbits $\{b\}$ over the ring $\mathbb{Z}_{p}$ on the set of primitive representations $Q[b]=A$, we deduce that all solutions $b$ constitute one orbit $\{b\}$. By (5), the orbit $\{C\}$ contains the representative $C=\left(\begin{array}{ll}0 & 0 \\ 1 & 0\end{array}\right)$, and then, by (8) , the form $Q$ decomposes into the following direct sum:

$$
Q=Q_{G}^{A}(C)=A_{1} \oplus\left(\begin{array}{cc}
A_{\geq p} & 1 \\
1 & 0
\end{array}\right) \oplus G_{a}
$$

where $J\left(A_{\geq p}\right)=\left(\begin{array}{rr}A_{\geq p} & 1 \\ 1 & 0\end{array}\right)$ is a hyperbolic form of dimension $2 m_{\geq p}, m_{\geq p}=\operatorname{dim} A_{\geq p}$. This means that the embedding $b: A \hookrightarrow Q$ splits into a direct orthogonal sum $b=$ $b_{1} \oplus b_{\geq p} \oplus b^{\perp}$ :

$$
b: A=A_{1} \oplus A_{\geq p} \hookrightarrow Q=A_{1} \oplus J\left(A_{\geq p}\right) \oplus Q^{\perp},
$$

where $Q^{\perp}=Q \ominus J(A)$ and $J(A)=A_{1} \oplus J\left(A_{\geq p}\right)$. Here the difference $X \ominus Y$ is any form $W$ in the decomposition $X \sim_{p} Y \oplus W$, which is determined uniquely (up to equivalence over $\mathbb{Z}_{p}$ ) for odd $p$, and nonuniquely in the general case for $p=2$. The existence of the difference of forms $X \ominus Y$ is equivalent to the condition $Y \leq X$. The embedding $b_{\text {min }}=b_{1} \oplus b_{\geq p}$ is the minimal embedding $b_{\text {min }}: A \hookrightarrow A_{1} \oplus J\left(A_{\geq p}\right)$ of the form $A$ of dimension $m_{1}+2 m_{\geq p}$. If we decompose the blocks $A_{1}$ and $A_{\geq p}$ into one-dimensional summands $A_{1}=\bigoplus_{i} A_{1, i}$ and $A_{\geq p}=\bigoplus_{j} A_{\geq p, j}$, then $b_{\min }$ decomposes into a direct sum of minimal indecomposable embeddings

$$
A_{1, i} \hookrightarrow A_{1, i}, \quad A_{\geq p, j} \hookrightarrow J\left(A_{\geq p, j}\right)
$$

of dimensions 1 or 2 , respectively.

From the decompositions (11) and (12) it follows that, up to equivalence over $\mathbb{Z}_{p}$, the form $G$ is uniquely determined by the forms $A$ and $Q$. For the form $G$, we have

$$
G=-a A_{\geq p}^{-1} \oplus a(Q \ominus J(A)) .
$$

The decomposition (12) implies the proposition below.

Proposition 1 (see [22]). If $p$ is odd and $p$ does not divide the determinant $|Q|$, then the form $A$ can be primitively represented over $\mathbb{Z}_{p}$ by the form $Q$ only if $J(A) \leq Q$. 
3.2. Now, let the odd prime $p$ divide $|Q|$, and let the form $Q$ have the following Jordan decomposition over $\mathbb{Z}_{p}$ :

$$
Q=Q_{1} \oplus p Q_{p}
$$

where the nonsingular blocks $\left|Q_{1}\right|$ and $\left|Q_{p}\right| \not \equiv 0(\bmod p), p \neq 2$, have dimensions $\operatorname{dim} Q_{1}=n-1$ and $\operatorname{dim} Q_{p}=1$ (i.e., the prime $p$ occurs in the determinant $|Q|$ to the first power). In this case, the set of primitive representations $b: Q[b]=A$ over the local ring $\mathbb{Z}_{p}$ splits into several distinct orbits $\{b\}$.

Theorem 3 (see [22]). For a form $Q=Q_{1} \oplus p Q_{p}, \operatorname{dim} Q_{p}=1$, the equation $Q[b]=A$ has a primitive solution if and only if at least one of the following forms exists:

$$
G^{\mathrm{I}}=-a A_{\geq p}^{-1} \oplus a\left(Q_{1} \ominus J(A)\right) \oplus a p Q_{p}
$$

or

$$
G^{\mathrm{II}}=-a\left(A_{\geq p} \ominus p Q_{p}\right)^{-1} \oplus a\left(Q_{1} \ominus\left(A_{1} \oplus J\left(m_{\geq p}-1\right)\right)\right),
$$

i.e., the inequalities $J(A) \leq Q_{1}$ or $A_{1} \oplus J\left(m_{\geq p}-1\right) \leq Q_{1}$ and $Q_{p} \leq A_{p}$ are fulfilled.

The block $J\left(m_{\geq p}-1\right)$ denotes the hyperbolic form $\left(\begin{array}{ll}0 & 1 \\ 1 & 0\end{array}\right)$ of dimension $2\left(m_{\geq p}-1\right)$, where $m_{\geq p}=\operatorname{dim} A_{\geq p}$.

In the notation of Subsection 3.1, let the form $A$ of dimension $m$ admit a decomposition $A=A_{1} \oplus A_{\geq p}$, where $A_{\geq p}=p A_{p} \oplus A_{>p}, A_{>p} \equiv 0\left(\bmod p^{2}\right)$, with blocks $A_{p}$ and $A_{\geq p}$ of dimension $m_{p}$ and $m_{\geq p}$. Consequently, if $n+1>m+m_{\geq p}$ and $m_{p}>1$, then the form $A$ is freely (i.e., in more than one way) primitively embedded in $Q$. Otherwise, $A$ is not embedded in $Q$ primitively, i.e., it is embedded rigidly. Theorem 3 easily implies the following sufficient conditions:

a first type embedding $b^{\mathrm{I}}: Q\left[b^{\mathrm{I}}\right]=A$ exists whenever

$$
\begin{aligned}
& n-1>m+m_{\geq p} ; \\
& n-1=m+m_{\geq p}, \quad \epsilon_{1}(Q)=\epsilon_{1}(A)\left(\frac{-1}{p}\right)^{m_{\geq p}} ;
\end{aligned}
$$

a second type embedding $b^{\mathrm{II}}: Q\left[b^{\mathrm{II}}\right]=A$ exists whenever

$$
\begin{aligned}
& n+1>m+m_{\geq p}, m_{p}>1 ; \\
& n+1=m+m_{\geq p}, m_{p}>1, \epsilon_{1}(Q)=\epsilon_{1}(A)\left(\frac{-1}{p}\right)^{m_{\geq p}-1} \\
& n+1>m+m_{\geq p}, m_{p}=1, \epsilon_{p}(Q)=\epsilon_{p}(A) ; \\
& n+1=m+m_{\geq p}, m_{p}=1, \epsilon_{1}(Q)=\epsilon_{1}(A)\left(\frac{-1}{p}\right)^{m_{\geq p}-1}, \epsilon_{p}(Q)=\epsilon_{p}(A) .
\end{aligned}
$$

Now we clarify the question as to when a quadratic form $Q$ in (15) of dimension 5 primitively represents all binary forms $A$ over $\mathbb{Z}_{p}$. Formulas (18) and (19) impose conditions on the shape and dimension of the blocks of $A$; for this reason it is necessary to consider six types of Jordan decompositions of binary forms $A$ over $\mathbb{Z}_{p}, p \neq 2$.

Type 1: $A=A_{1}, \operatorname{dim} A_{1}=2$.

The form $G^{\mathrm{I}}$ exists, because condition (18,1) is satisfied. Using formula (16), we deduce that $G^{\mathrm{I}}=\left(Q_{1} \ominus A\right) \oplus p Q_{p}$ and $p_{G^{\mathrm{I}}}=1^{\epsilon_{1}(Q) \epsilon_{1}(A) 2} p^{\epsilon_{p}(Q) 1}$, where $p_{A}=1^{\epsilon_{1}(A) 1}$.

The form $G^{\mathrm{II}}$ does not exist, because none of conditions (19) is fulfilled.

Type 2: $A=p A_{p}, \operatorname{dim} A_{p}=2$.

The binary form $A=p A_{p}$ of level $a=p$ has the $p$-symbol $p_{A}=p^{\epsilon_{p}(A) 2}$. From formula (18,2), we derive that the form $G^{\mathrm{I}}$ exists if $\epsilon_{1}(Q)=\left(\frac{-1}{p}\right)^{2}=1$. Using formula (16), we obtain $G^{\mathrm{I}}=-p\left(p A_{p}\right)^{-1} \oplus p\left(Q_{1} \ominus J\left(p A_{p}\right)\right) \oplus p^{2} Q_{p}=-A_{p}^{-1} \oplus p^{2} Q_{p} \quad$ (if $\left.\epsilon_{1}(Q)=1\right)$ and $p_{G^{\mathrm{I}}}=1^{\left(\frac{-1}{p}\right)^{2} \epsilon_{p}(A) 2}\left(p^{2}\right)^{\epsilon_{p}(Q) 1}$. 
By (19) 1$)$, the form $G^{\mathrm{II}}$ exists. By (17), we have $G^{\mathrm{II}}=-p\left(p A_{p} \ominus p Q_{p}\right)^{-1} \oplus p\left(Q_{1} \ominus\right.$

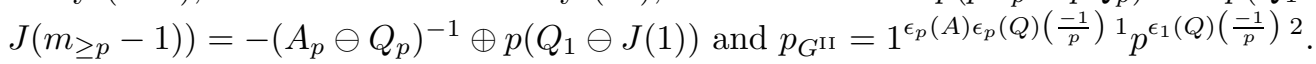

Type 3: $A=p^{\alpha_{1}} A_{p^{\alpha_{1}}} \oplus p^{\alpha_{2}} A_{p^{\alpha_{2}}}, 2 \leq \alpha_{1} \leq \alpha_{2}$.

The binary form $A$ has the $p$-symbol $p_{A}=\left(p^{\alpha_{1}}\right)^{\epsilon_{p^{\alpha_{1}}}(A){ }^{1}}\left(p^{\alpha_{2}}\right)^{\epsilon_{p^{\alpha_{2}}}(A) 1}$ and $a=p^{\alpha_{2}}$. From (18,2), we see that the form $G^{\mathrm{I}}$ exists if $\epsilon_{1}(Q)=\left(\frac{-1}{p}\right)^{2}=1$. By (16), we have $G^{\mathrm{I}}=-p^{\alpha_{2}}\left(p^{\alpha_{1}} A_{p^{\alpha_{1}}} \oplus p^{\alpha_{2}} A_{p^{\alpha_{2}}}\right)^{-1} \oplus p^{\alpha_{2}}\left(Q_{1} \ominus J\left(p^{\alpha_{1}} A_{p^{\alpha_{1}}}\right) \ominus J\left(p^{\alpha_{2}} A_{p^{\alpha_{2}}}\right)\right) \oplus p^{\alpha_{2}} p Q_{p}=$ $-\left(p^{\alpha_{2}-\alpha_{1}} A_{p^{\alpha_{1}}}^{-1} \oplus A_{p^{\alpha_{2}}}^{-1}\right) \oplus p^{\alpha_{2}+1} Q_{p}\left(\right.$ if $\left.\epsilon_{1}(Q)=1\right)$ and

$$
p_{G^{\mathrm{I}}}=1^{\epsilon_{p^{\alpha_{2}}}(A)\left(\frac{-1}{p}\right) 1}\left(p^{\alpha_{2}-\alpha_{1}}\right)^{\epsilon_{p^{\alpha_{1}}}(A)\left(\frac{-1}{p}\right){ }^{1}}\left(p^{\alpha_{2}+1}\right)^{\epsilon_{p}(Q) 1} .
$$

The form $G^{\mathrm{II}}$ does not exist, because none of conditions (19) is fulfilled.

Type 4: $A=A_{1} \oplus p^{\alpha_{1}} A_{p^{\alpha_{1}}}, \alpha_{1} \geq 2, \operatorname{dim} A_{1}=\operatorname{dim} A_{p^{\alpha_{1}}}=1$.

The binary form $A$ of level $a=p^{\alpha_{1}}$ has the $p$-symbol $p_{A}=1^{\epsilon_{1}(A){ }^{1}}\left(p^{\alpha_{1}}\right)^{\epsilon_{p} \alpha_{1}}(A){ }^{1}$. By (18,1), the form $G^{\mathrm{I}}$ exists. By (16), we have $G^{\mathrm{I}}=-p^{\alpha_{1}}\left(p^{\alpha_{1}} A_{p^{\alpha_{1}}}\right)^{-1} \oplus p^{\alpha_{1}}\left(Q_{1} \ominus\left(A_{1} \oplus\right.\right.$ $\left.\left.J\left(p^{\alpha_{1}} A_{p^{\alpha_{1}}}\right)\right) \oplus p Q_{p}\right)=-A_{p^{\alpha_{1}}}^{-1} \oplus p^{\alpha_{1}}\left(Q_{1} \ominus\left(A_{1} \oplus J\left(p^{\alpha_{1}} A_{p^{\alpha_{1}}}\right)\right)\right) \oplus p^{\alpha_{1}+1} Q_{p}$, and the $p$-adic symbol of the form $G^{\mathrm{I}}$ is equal to $1^{\epsilon_{p} \alpha_{1}(A)\left(\frac{-1}{p}\right){ }^{1}}\left(p^{\alpha_{1}}\right)^{\epsilon_{1}(A) \epsilon_{1}(Q)\left(\frac{-1}{p}\right){ }^{1}\left(p^{\alpha_{1}+1}\right)^{\epsilon_{p}}(Q) 1}$.

The form $G^{\mathrm{II}}$ does not exist, because none of conditions (19) is fulfilled.

Type 5: $A=A_{1} \oplus p A_{p}, \operatorname{dim} A_{1}=\operatorname{dim} A_{p}=1$.

The binary form $A$ of step $a=p$ has the $p$-symbol $p_{A}=1^{\epsilon_{1}(A) 1} p^{\epsilon_{p}(A) 1}$. By (18) 1 ), the form $G^{\mathrm{I}}$ exists. By (16), we have $G^{\mathrm{I}}=-p\left(p A_{p}\right)^{-1} \oplus p\left(Q_{1} \ominus\left(A_{1} \oplus J\left(p A_{p}\right)\right) \oplus p Q_{p}\right)=$

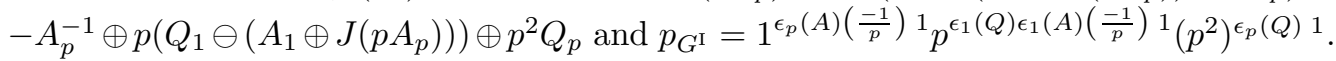

From (19,3) we derive that the form $G^{\mathrm{II}}$ exists if $\epsilon_{p}(Q)=\epsilon_{p}(A)$. By (17), we have $G^{\mathrm{II}}=-p\left(p A_{p} \ominus p Q_{p}\right)^{-1} \oplus p\left(Q_{1} \ominus A_{1}\right)=-\left(A_{p} \ominus Q_{p}\right)^{-1} \oplus p\left(Q_{1} \ominus A_{1}\right)=p\left(Q_{1} \ominus A_{1}\right)$ (if $\left.\epsilon_{p}(Q)=\epsilon_{p}(A)\right)$ and $p_{G^{\mathrm{II}}}=p^{\epsilon_{1}(A) \epsilon_{1}(Q) 3}$.

Type 6: $A=p A_{p} \oplus p^{\alpha_{1}} A_{p^{\alpha_{1}}}, \alpha_{1} \geq 2, \operatorname{dim} A_{p}=\operatorname{dim} A_{p^{\alpha_{1}}}=1$.

The binary form $A$ of level $a=p^{\alpha_{1}}$ has the $p$-symbol $p_{A}=p^{\epsilon_{p}(A){ }^{1}}\left(p^{\alpha_{1}}\right)^{\epsilon_{p} \alpha_{1}}(A) 1$. From (18,2) we conclude that the form $G^{\mathrm{I}}$ exists if $\epsilon_{1}(Q)=\left(\frac{-1}{p}\right)^{2}=1$. By (16)), we have $G^{\mathrm{I}}=-p^{\alpha_{1}}\left(p A_{p} \oplus p^{\alpha_{1}} A_{p^{\alpha_{1}}}\right)^{-1} \oplus p^{\alpha_{1}}\left(Q_{1} \ominus J\left(p A_{p}\right) \ominus J\left(p^{\alpha_{1}} A_{p^{\alpha_{1}}}\right) \oplus p Q_{p}\right)=-\left(p^{\alpha_{1}-1} A_{p}^{-1} \oplus\right.$ $\left.A_{p^{\alpha_{1}}}^{-1}\right) \oplus p^{\alpha_{1}+1} Q_{p}$ (if $\left.\epsilon_{1}(Q)=1\right)$ and $p_{G^{\mathrm{I}}}=1^{\epsilon_{p^{\alpha_{1}}}(A)\left(\frac{-1}{p}\right){ }^{1}}\left(p^{\alpha_{1}-1}\right)^{\epsilon_{p}(A)\left(\frac{-1}{p}\right){ }^{1}}\left(p^{\alpha_{1}+1}\right)^{\epsilon_{p}(Q) 1}$.

By (19.3), the form $G^{\mathrm{II}}$ exists if $\epsilon_{p}(Q)=\epsilon_{p}(A)$. From (17) we get $G^{\mathrm{II}}=-p^{\alpha_{1}}\left(p A_{p} \oplus\right.$ $\left.p^{\alpha_{1}} A_{p^{\alpha_{1}}} \ominus p Q_{p}\right)^{-1} \oplus p^{\alpha_{1}}\left(Q_{1} \ominus J(1)\right)=-\left(A_{p^{\alpha_{1}}}^{-1} \oplus p^{\alpha_{1}-1}\left(A_{p} \ominus Q_{p}\right)\right) \oplus p^{\alpha_{1}}\left(Q_{1} \ominus J(1)\right)=$ $-\left(A_{p^{\alpha_{1}}}^{-1} \oplus p^{\alpha_{1}}\left(Q_{1} \ominus J(1)\right)\right.$ (if $\left.\epsilon_{p}(Q)=\epsilon_{p}(A)\right)$ and $p_{G^{\mathrm{II}}}=1^{\epsilon_{p^{\alpha_{1}}}(A) 1}{ }^{1}\left(p^{\alpha_{1}}\right)^{\epsilon_{1}(Q)\left(\frac{-1}{p}\right) 2}$.

In the table below, we collect the above existence conditions for the forms $G^{\mathrm{I}}$ and $G^{\mathrm{II}}$, obtained when embedding the binary forms $A$ in forms $Q$ as in (15) of dimension 5 .

The said above allows us to state the following proposition.

Proposition 2. A quadratic form $Q=Q_{1} \oplus p Q_{p}$, $\operatorname{dim} Q_{p}=1$, of dimension 5 is primitively $p \sim$ universal, $p \neq 2$, if and only if $\epsilon_{1}(Q)=1$.

Similarly, we obtain Table 2, which contains embeddings of binary forms $A$ in forms $Q$ as in (15) of dimension $n \geq 3(n \neq 5)$ over $\mathbb{Z}_{p}$. The sign $+/-$ denotes the presence/absence of an embedding of forms $A$, and the sign $+_{i}, i=1,2,3,4$, corresponds to the existence of an embedding of forms $A$ under the condition $\epsilon_{1}(Q)=\epsilon_{1}(A), \epsilon_{1}(Q)=$ $\left(\frac{-1}{p}\right), \epsilon_{p}(Q)=\epsilon_{p}(A)$, and $\epsilon_{1}(Q)=\epsilon_{1}(A)\left(\frac{-1}{p}\right)$, respectively.

In accordance with Table 2 , the forms $Q$ in (15) are not primitively $p \sim$ universal for $n=3,4$, while for $n \geq 6$ the forms $Q$ are primitively $p \sim$ universal without any conditions on their Jordan constituents. 
TABLE 1

\begin{tabular}{|c|c|c|}
\hline$A$ & $G^{\mathrm{I}}$ & $G^{\mathrm{II}}$ \\
\hline$A_{1}$ & + & - \\
\hline$p A_{p}$ & + for $\epsilon_{1}(Q)=1$ & + \\
\hline$p^{\alpha_{1}} A_{p^{\alpha_{1}} \oplus p^{\alpha_{2}} A_{p^{\alpha_{2}}}, 2 \leq \alpha_{1} \leq \alpha_{2}}+$ for $\epsilon_{1}(Q)=1$ & - \\
\hline$A_{1} \oplus p^{\alpha_{1}} A_{p^{\alpha_{1}}}, \alpha_{1} \geq 2$ & + & - \\
\hline$A_{1} \oplus p A_{p}$ & + & + for $\epsilon_{p}(Q)=\epsilon_{p}(A)$ \\
\hline$p A_{p} \oplus p^{\alpha_{1}} A_{p^{\alpha_{1}}}, \alpha_{1} \geq 2$ & + for $\epsilon_{1}(Q)=1$ & + for $\epsilon_{p}(Q)=\epsilon_{p}(A)$ \\
\hline
\end{tabular}

TABLE 2

\begin{tabular}{|c|c|c|c|c|c|c|c|}
\hline$n$ & & Type 1 & Type 2 & Type 3 & Type 4 & Type 5 & Type 6 \\
\hline 3 & $G^{\mathrm{I}}$ & $+_{1}$ & - & - & - & - & - \\
\cline { 2 - 8 } & $G^{\mathrm{II}}$ & - & $+_{2}$ & - & - & $+_{3}$ & $+_{2},+_{3}$ \\
\hline \multirow{2}{*}{4} & $G^{\mathrm{I}}$ & + & - & - & $+_{4}$ & $+_{4}$ & - \\
\cline { 2 - 8 } & $G^{\mathrm{II}}$ & - & + & - & - & $+_{3}$ & $+_{3}$ \\
\hline \multirow{2}{*}{$\geq 6$} & $G^{\mathrm{I}}$ & + & + & + & + & + & + \\
\cline { 2 - 8 } & $G^{\mathrm{II}}$ & - & + & - & - & $++_{3}$ & $++_{3}$ \\
\hline
\end{tabular}

The analysis of Tables 1 and 2 yields the following result.

Theorem 4. A form $Q=Q_{1} \oplus p Q_{p}$ such that $\operatorname{dim} Q=n$ and $\operatorname{dim} Q_{p}=1$ is primitively $p \sim$ universal, $p \neq 2$, if one of the following conditions is fulfilled:

1) $n=5$ and $\epsilon_{1}(Q)=+1$;

2) $n \geq 6$.

\section{§4. Primitive Representations of forms over $\mathbb{Z}_{2}$}

Primitive representations of $A$ by a form $Q$ over the even ring $\mathbb{Z}_{2}$ begin to ramify and depend heavily on the invariants of the forms $A$ and $Q$. We want to find existence conditions for primitive representations of binary forms $A$.

4.1. The local classification of the quadratic forms $Q$ over $\mathbb{Z}_{2}$ is significantly more complicated. This is explained by the presence of two additional invariants, which leads to the necessity of modifying the 2 -adic symbols. Over $\mathbb{Z}_{2}$, a nondegenerate form $Q \in M_{n}\left(\mathbb{Z}_{2}\right)$ has a Jordan decomposition $Q=\sum_{q} q Q_{q}$ with scales $q=2^{\alpha}, \alpha=0,1,2, \ldots$, and $Q_{q} \in G L\left(\mathbb{Z}_{2}\right)$. The $p$-adic symbol is

$$
2_{Q}=\prod_{q} q_{\xi_{q}(Q) t_{q}(Q)}^{\epsilon_{q}(Q) n_{q}(Q)}
$$

(see [23]); moreover, $n_{q}(Q)=\operatorname{dim} Q_{q}$ and $\epsilon_{q}(Q)=\left(\frac{|Q|}{2}\right)$ are, respectively, the dimension and the sign of the Jordan constituent $q Q_{q}$, where

$$
\left(\frac{a}{2}\right)=\left\{\begin{array}{lll}
+1, & a \equiv \pm 1 \quad(\bmod 8), \\
-1, & a \equiv \pm 3 \quad(\bmod 8)
\end{array}\right.
$$


is the Jacobi-Legendre symbol; the symbol $\xi_{q}(Q)=I$, called the type of $Q_{q}$, takes the values I or II for odd and even forms $Q_{q}$, respectively; $t_{q}(Q)$ is called the oddity of $Q_{q}$ and is determined modulo 8. Existence conditions for $q Q_{q}$ were given in [23].

Let the determinant of the form $Q$ be an odd number, and let

$$
Q=Q_{G}^{A}(C)=\frac{1}{a}\left(\begin{array}{cc}
a A & 0 \\
0 & G
\end{array}\right)\left[M_{C}^{-1}\right]=\left(\begin{array}{cc}
A & C \\
{ }^{t} C & Q^{\prime}
\end{array}\right),
$$

where $a=2^{\nu}$ is the level of the form $A$, and the symmetric matrix $Q^{\prime}$ satisfies the congruence

$$
Q^{\prime}=A^{-1}[C]+\frac{1}{a} G \equiv 0 \quad\left(\bmod \mathbb{Z}_{p}\right) \quad \text { or } \quad Q^{\prime} \doteq 0 \quad\left(\bmod \mathbb{Z}_{p}\right)
$$

if the matrix $Q^{\prime}$ is odd or even, respectively. The congruence $\doteq$ means that $Q^{\prime} \in M_{k}\left(\mathbb{Z}_{p}\right)$ is an even matrix, i.e., it has an even diagonal.

Over the ring $\mathbb{Z}_{p}=\mathbb{Z}_{2}$, a quadratic form reduces as follows:

$$
A \sim{ }_{2} \operatorname{diag}\left(p^{\nu_{1}} A_{1}, \ldots, p^{\nu_{k}} A_{k}\right), \quad 0 \leq \nu_{1}<\cdots<\nu_{k}, \quad A_{i} \in G L\left(\mathbb{Z}_{p}\right)
$$

(see [23]). For this reason, we may assume that $A=\left(\begin{array}{cc}A_{1} & 0 \\ 0 & A_{\geq p}\end{array}\right)$, where $A_{1} \in G L\left(\mathbb{Z}_{p}\right)$ is of maximal dimension, and the conditions

$$
m_{p} \leq k=\operatorname{dim} G, \quad C_{p}=\left(1_{m_{p}} 0\right) U, \quad U \in G L_{k}\left(\mathbb{Z}_{p}\right),
$$

are satisfied for $p=2$ and odd $|Q|$.

In the congruence (5), $C \bmod A$ can be taken in the form $\left(\begin{array}{c}0 \\ C\end{array}\right)$. Using (22), we obtain

$$
Q=\left(\begin{array}{cc}
A & C \\
{ }^{t} C & Q^{\prime}
\end{array}\right)=A_{1} \oplus\left(\begin{array}{cc}
A_{\geq p} & C \\
{ }^{t} C & Q^{\prime},
\end{array}\right)
$$

where $Q^{\prime}=(E[C]+G) / a$ and $E=a A_{\geq p}^{-1}$. In particular, if $A=A_{1}$, then (26) implies that

$$
G=G_{1}=Q \ominus A_{1} .
$$

By (26), it suffices to consider the form $A=A_{\geq p}$, i.e., $A \equiv 0(\bmod 2)$. Using (15) and (25), we obtain

$$
\frac{1}{a} G \stackrel{*}{\equiv}-\left(\begin{array}{cc}
A_{\geq p}^{-1} & 0 \\
0 & 0
\end{array}\right)[U] \quad\left(\bmod \mathbb{Z}_{2}\right) .
$$

By (28), up to equivalence over $G L\left(\mathbb{Z}_{2}\right)$, we have the matrix congruence

$$
G \stackrel{*}{\equiv}\left(\begin{array}{cc}
-E & 0 \\
0 & 0
\end{array}\right) \quad(\bmod a)
$$

where the symbol $\stackrel{*}{\equiv}$ means $\equiv$ for odd forms $Q=Q_{\mathrm{I}}$ and $\doteq$ for even forms $Q=Q_{\mathrm{II}}$.

4.2. Consider the one-dimensional case: $A=q A_{q}, \operatorname{dim} A_{q}=1$, with step $a=q=2^{\alpha}$, $\alpha \geq 1$ (see 25]). The congruence (29) means that

$$
G=G_{1} \oplus q G_{q}, \quad \operatorname{dim} G_{1}=1, \quad G_{1} \equiv-A_{q} \quad(\bmod q) .
$$

In (5) we take $C=\left(C_{1}|0 \ldots| 0\right)$, where the number $C_{1}$ satisfies the condition $C_{1}^{2} \equiv 1$ $(\bmod q)$ and

$$
C_{1}= \begin{cases}1 & \text { for } \alpha=1 \\ \pm 1 & \text { for } \alpha=2 \\ \pm 1, \pm\left(1+2^{\alpha-1}\right) & \text { for } \alpha \geq 3\end{cases}
$$


By (26), we have $Q=J\left(q A_{q}\right) \oplus G_{q}$, where the embedding $J\left(q A_{q}\right)$ is minimal for the form $A=q A_{q}, \operatorname{dim} A_{q}=1$. From (30) and the previous decomposition, it follows that

$$
G=G_{1} \oplus q\left(Q \ominus J\left(q A_{q}\right)\right),
$$

where the minimal embeddings $J\left(q A_{q}\right)$ of dimension 2 are as follows:

$$
J\left(q A_{q}\right)= \begin{cases}\left(\begin{array}{cc}
q A_{q} & 1 \\
1 & \left(A_{q}+G_{1}\right) / q
\end{array}\right) & \text { for } \alpha=1,2, \\
\left(\begin{array}{cc}
q A_{q} & 1 \\
1 & 0
\end{array}\right)_{\mathrm{II}},\left(\begin{array}{cc}
q A_{q} & 1+q / 2 \\
1+q / 2 & A_{q}(1+q / 4)
\end{array}\right)_{\mathrm{I}} & \text { for } \alpha \geq 3 .\end{cases}
$$

Straightforward calculations yield all invariants of the forms $J=J\left(q A_{q}\right)$ :

$$
\text { for } \alpha=1 \text { : }
$$

$$
\begin{aligned}
& |J| \equiv A_{2} G_{1} \quad(\bmod 8), \\
& J=J_{\mathrm{II}} \text { if } A_{2}+G_{1} \equiv 0 \quad(\bmod 4), \quad t\left(J_{\mathrm{II}}\right)=0, \\
& J=J_{\mathrm{I}} \text { if } A_{2}+G_{1} \not \equiv 0 \quad(\bmod 4), \\
& t\left(J_{\mathrm{I}}\right) \equiv\left(A_{2}+G_{1}\right)\left(1+A_{2} G_{1}\right) / 2 \quad(\bmod 8) ;
\end{aligned}
$$

for $\alpha=2$ :

for $\alpha \geq 3$

$$
\begin{aligned}
& |J| \equiv A_{4} G_{1} \quad(\bmod 8) \\
& J=J_{\mathrm{II}} \quad \text { if } A_{4}+G_{1} \equiv 0 \quad(\bmod 8), \quad t\left(J_{\mathrm{II}}\right)=0 \\
& J=J_{\mathrm{I}} \text { if } A_{4}+G_{1} \not \equiv 0 \quad(\bmod 8) \\
& t\left(J_{\mathrm{I}}\right) \equiv A_{4}+G_{1} \quad(\bmod 8)
\end{aligned}
$$

$$
\left|J\left(q A_{q}\right)\right| \equiv-1, \quad t\left(J\left(q A_{q}\right)\right) \equiv 0 \quad(\bmod 8) .
$$

By (26), the general forms $A=A_{1} \oplus q A_{q}, \operatorname{dim} A_{q}=1$, give the decomposition

$$
G=G_{1} \oplus q(Q \ominus J(A)),
$$

where $J(A)=A_{1} \oplus J\left(q A_{q}\right)$.

4.3. First, consider the forms $A=q A_{q}$ with an odd block $A_{q}$ of dimension 2 . The local classification over $\mathbb{Z}_{2}$ implies that $A_{q}$ is one of the following six diagonal forms:

$$
\begin{aligned}
& 0^{+}=\left(\begin{array}{ll}
1 & \\
& 7
\end{array}\right), 2^{+}=\left(\begin{array}{ll}
1 & \\
& 1
\end{array}\right), 6^{+}=\left(\begin{array}{ll}
3 & \\
& 3
\end{array}\right), \\
& 2^{-}=\left(\begin{array}{ll}
3 & \\
& 7
\end{array}\right), 4^{-}=\left(\begin{array}{ll}
1 & \\
& 3
\end{array}\right), 6^{-}=\left(\begin{array}{ll}
1 & \\
& 5
\end{array}\right) .
\end{aligned}
$$

The signs + and - denote the sign of the determinant, and the number of a form denotes its oddity $t$.

The congruences $G \equiv^{*}\left(\begin{array}{cc}-E & 0 \\ 0 & 0\end{array}\right)(\bmod q)$ impose the following restrictions on $G$ : $G=$ $G_{1} \oplus q G_{q}, G_{1} \equiv-A_{q}(\bmod q), \operatorname{dim} G_{1}=2$. For this reason, $G_{1}$ is one of the forms

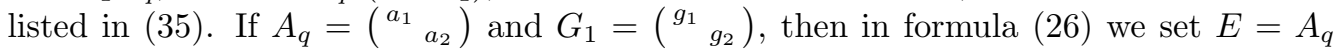
and $C=\left(C_{1} 0\right)$, where the $(2 \times 2)$-matrix $C_{1}$ is a solution of the matrix congruence $A_{q}\left[C_{1}\right] \equiv-G_{1}(\bmod q)$. 
The case where $q=2$ was studied in the paper [25], where the minimal decomposable embeddings

$$
\begin{aligned}
& J\left(2 A_{2}\right)=\left(\begin{array}{cc}
2 a_{1} & 1 \\
1 & \left(a_{1}+g_{1}\right) / 2
\end{array}\right) \oplus\left(\begin{array}{cc}
2 a_{2} & 1 \\
1 & \left(a_{2}+g_{2}\right) / 2
\end{array}\right) \quad \text { or } \\
& J\left(2 A_{2}\right)=\left(\begin{array}{cc}
2 a_{1} & 1 \\
1 & \left(a_{1}+g_{2}\right) / 2
\end{array}\right) \oplus\left(\begin{array}{cc}
2 a_{2} & 1 \\
1 & \left(a_{2}+g_{1}\right) / 2
\end{array}\right)
\end{aligned}
$$

in the form $Q$ of odd determinant were described for the binary forms $A=2 A_{2}$ with an odd block $A_{2}$.

Next, let $q=4$. The group $S L_{2}(\mathbb{Z} / 4 \mathbb{Z})$ contains 48 elements. Searching through these elements, we see that the congruence $A_{4}\left[C_{1}\right] \equiv-G_{1}(\bmod 4)$ is true only for the following matrices $C_{1}$ :

$$
\left(\begin{array}{cc} 
\pm 1 & 0 \\
0 & \pm 1
\end{array}\right),\left(\begin{array}{cc} 
\pm 1 & 2 \\
2 & \pm 1
\end{array}\right),\left(\begin{array}{cc}
0 & \mp 1 \\
\pm 1 & 0
\end{array}\right),\left(\begin{array}{cc}
2 & \pm 1 \\
\mp 1 & 2
\end{array}\right)
$$

which form a group isomorphic to the group $C_{2} \times C_{4}$. Here $C_{k}$ is the cyclic group of order $k$.

By (26), the form $Q$ decomposes as the follows:

$$
Q=\left(\begin{array}{cc}
4 A_{4} & C_{1} \\
{ }^{t} C_{1} & \left(A_{4}\left[C_{1}\right]+G_{1}\right) / 4
\end{array}\right) \oplus G_{4}=J\left(4 A_{4}\right) \oplus G_{4}
$$

with the embedding $J=J\left(4 A_{4}\right)$ minimal for the form $4 A_{4}$. We describe the blocks $J\left(4 A_{4}\right)$ over $\mathbb{Z}_{2}$ for each of the matrices $C_{1}$ in (37).

For $C_{1}^{(1)}$ we have $J\left(4 A_{4}\right)=\left(\begin{array}{cc}4 a_{1} & \pm 1 \\ \pm 1 & \left(a_{1}+g_{1}\right) / 4\end{array}\right) \oplus\left(\begin{array}{cc}4 a_{2} & \pm 1 \\ \pm 1 & \left(a_{2}+g_{2}\right) / 4\end{array}\right)$. Direct calculation yields all invariants of the form $J\left(4 A_{4}\right)=J_{1} \oplus J_{2}$ :

$$
\begin{aligned}
\left|J\left(4 A_{4}\right)\right| & \equiv a_{1} a_{2} g_{1} g_{2} \quad(\bmod 8), \quad\left|J_{i}\right| \equiv a_{i} g_{i} \quad(\bmod 8), \quad i=1,2 ; \\
J_{i} & =J_{\mathrm{II}}, t\left(J_{i}\right)=0 \text { if } a_{i}+g_{i} \equiv 0 \quad(\bmod 8) ; \\
J_{i} & =J_{\mathrm{I}}, t\left(J_{i}\right) \equiv a_{i}+g_{i}(\bmod 8) \equiv 4(\bmod 8) \text { if } a_{i}+g_{i} \equiv 4(\bmod 8) .
\end{aligned}
$$

Consequently, the 2-symbol of the form $J\left(4 A_{4}\right)$ can look like this:

$$
2_{J\left(4 A_{4}\right)}= \begin{cases}1_{\mathrm{I}, 0}^{\epsilon 4} & \text { if } a_{1}+g_{1} \equiv 4 \quad(\bmod 8) \text { and } a_{2}+g_{2} \equiv 4 \quad(\bmod 8), \\ 1_{\mathrm{II}, 0}^{\epsilon} & \text { if } a_{1}+g_{1} \equiv 0 \quad(\bmod 8) \text { and } a_{2}+g_{2} \equiv 0 \quad(\bmod 8), \\ 1_{\mathrm{I}, 4}^{\epsilon 4} & \text { if } a_{1}+g_{1} \equiv 4(0) \quad(\bmod 8) \text { and } a_{2}+g_{2} \equiv 0(4) \quad(\bmod 8),\end{cases}
$$

where $\epsilon=\left(\frac{a_{1} a_{2} g_{1} g_{2}}{2}\right)$.

For $C_{1}^{(2)}$ we obtain

$$
J\left(4 A_{4}\right)=\left(\begin{array}{cccc}
4 a_{1} & 0 & \pm 1 & 2 \\
0 & 4 a_{2} & 2 & \pm 1 \\
\pm 1 & 2 & a_{2}+\frac{a_{1}+g_{1}}{4} & \pm \frac{a_{1}+a_{2}}{2} \\
2 & \pm 1 & \pm \frac{a_{1}+a_{2}}{2} & a_{1}+\frac{a_{2}+g_{2}}{4}
\end{array}\right)
$$

and $\left|J\left(4 A_{4}\right)\right| \equiv\left|A_{4}\right|\left|G_{1}\right| \equiv a_{1} a_{2} g_{1} g_{2}(\bmod 8)$.

Using the matrix analog of the reduction of quadratic forms to a diagonal form over the local ring $\mathbb{Z}_{2}$ (see $\left[20,23\right.$ ), we see that the form $J\left(4 A_{4}\right)$ over $\mathbb{Z}_{2}$ is equivalent to the form

$$
\left(\begin{array}{cc}
4 a_{1} & 1 \\
1 & a_{2}+\frac{a_{1}+g_{1}}{4}
\end{array}\right) \oplus\left(\begin{array}{cc}
4 a_{2} & 1+4 a_{1} g_{1} \\
1+4 a_{1} g_{1} & g_{1}+4 g_{2}+2 a_{2} g_{1} g_{2}+\frac{a_{2}+g_{2}}{4}
\end{array}\right)=J_{1} \oplus J_{2},
$$

where $\left|J_{1}\right| \equiv a_{1}\left(4 a_{2}+g_{1}\right)(\bmod 8)$ and $\left|J_{2}\right| \equiv a_{2}\left(4 g_{1}+g_{2}\right)(\bmod 8)$. 
Direct calculation provides all invariants of the form $J\left(4 A_{4}\right)$ :

$$
\begin{aligned}
& J_{i}=J_{\mathrm{II}}, t\left(J_{i}\right)=0, i=1,2, \text { if } a_{i}+g_{i} \equiv 0 \quad(\bmod 8) ; \\
& J_{1}=J_{\mathrm{I}}, t\left(J_{1}\right) \equiv\left(1+a_{1} g_{1}\right)\left(\frac{a_{1}+g_{1}}{4}+2 a_{2}\right)+4 a_{1} \equiv 4 a_{1}(\bmod 8) \equiv 4(\bmod 8) \\
& \quad \text { if } a_{1}+g_{1} \equiv 4 \quad(\bmod 8) ; \\
& J_{2}=J_{\mathrm{I}}, t\left(J_{2}\right) \equiv\left(1+a_{2} g_{2}\right) \frac{a_{2}+g_{2}}{4}+4 a_{2} g_{1} g_{2} \equiv 4 a_{2} g_{1} g_{2}(\bmod 8) \equiv 4(\bmod 8) \\
& \quad \text { if } a_{2}+g_{2} \equiv 4 \quad(\bmod 8) .
\end{aligned}
$$

Calculation of the 2-symbols of the form $J\left(4 A_{4}\right)$ yield formulas (39).

For $C_{1}^{(3)}=\left(\begin{array}{cc}0 & \mp 1 \\ \pm 1 & 0\end{array}\right)$ and $a_{1} \equiv a_{2}(\bmod 4)$, we deduce that the block $J\left(4 A_{4}\right)$ can be represented as $\left(\begin{array}{cc}4 a_{1} & \pm 1 \\ \pm 1 & \left(a_{1}+g_{2}\right) / 4\end{array}\right) \oplus\left(\begin{array}{cc}4 a_{2} & \mp 1 \\ \mp 1 & \left(a_{2}+g_{1}\right) / 4\end{array}\right)$. In this case the 2 -symbols of the form $J\left(4 A_{4}\right)$ are calculated similarly:

(40) $2_{J\left(4 A_{4}\right)}=\left\{\begin{array}{llll}1_{\mathrm{I}, 0}^{\epsilon 4} & \text { if } a_{1}+g_{2} \equiv 4 \quad(\bmod 8) \quad \text { and } a_{2}+g_{1} \equiv 4 \quad(\bmod 8), \\ 1_{\mathrm{II}, 0}^{\epsilon 4} & \text { if } a_{1}+g_{2} \equiv 0 \quad(\bmod 8) \quad \text { and } a_{2}+g_{1} \equiv 0 \quad(\bmod 8), \\ 1_{\mathrm{I}, 4}^{\epsilon 4} & \text { if } a_{1}+g_{2} \equiv 4(0) \quad(\bmod 8) \quad \text { and } a_{2}+g_{1} \equiv 0(4) \quad(\bmod 8),\end{array}\right.$

where $\epsilon=\left(\frac{a_{1} a_{2} g_{1} g_{2}}{2}\right)$.

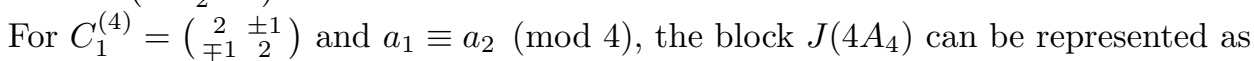

$$
\left(\begin{array}{cccc}
4 a_{1} & 0 & 2 & \pm 1 \\
0 & 4 a_{2} & \mp 1 & 2 \\
2 & \mp 1 & a_{1}+\frac{a_{2}+g_{1}}{4} & \pm \frac{a_{1}-a_{2}}{2} \\
\pm 1 & 2 & \pm \frac{a_{1}-a_{2}}{2} & a_{2}+\frac{a_{1}+g_{2}}{4}
\end{array}\right)
$$

and $\left|J\left(4 A_{4}\right)\right| \equiv\left|A_{4}\right|\left|G_{1}\right| \equiv a_{1} a_{2} g_{1} g_{2}(\bmod 8)$. Over $\mathbb{Z}_{2}$ the form $J\left(4 A_{4}\right)$ is equivalent to the following direct sum of forms:

$$
\left(\begin{array}{cc}
4 a_{1} & 1 \\
1 & a_{2}+\frac{a_{1}+g_{2}}{4}
\end{array}\right) \oplus\left(\begin{array}{cc}
4 a_{2} & -1+4 a_{1} g_{2} \\
-1+4 a_{1} g_{2} & 4 g_{1}+g_{2}+2 a_{2} g_{1} g_{2}+\frac{a_{2}+g_{1}}{4}
\end{array}\right),
$$

where $\left|J_{1}\right| \equiv a_{1}\left(4 a_{2}+g_{2}\right)(\bmod 8)$ and $\left|J_{2}\right| \equiv a_{2}\left(4 g_{2}+g_{1}\right)(\bmod 8)$. Straightforward calculations yield all invariants of the form $J\left(4 A_{4}\right)$ :

$$
\begin{aligned}
& J_{1}=J_{\mathrm{II}}, t\left(J_{1}\right)=0 \text { if } a_{1}+g_{2} \equiv 0 \quad(\bmod 8) ; \\
& J_{2}=J_{\mathrm{II}}, t\left(J_{2}\right)=0 \text { if } a_{2}+g_{1} \equiv 0 \quad(\bmod 8) ; \\
& J_{1}=J_{\mathrm{I}}, t\left(J_{1}\right) \equiv\left(1+a_{1} g_{2}\right)\left(\frac{a_{1}+g_{2}}{4}+2 a_{2}\right)+4 a_{1} \equiv 4 a_{1}(\bmod 8) \equiv 4(\bmod 8) \\
& \quad \text { if } a_{1}+g_{2} \equiv 4 \quad(\bmod 8) ; \\
& J_{2}=J_{\mathrm{I}}, t\left(J_{2}\right) \equiv\left(1+a_{2} g_{1}\right) \frac{a_{2}+g_{1}}{4}+4 a_{2} g_{1} g_{2} \equiv 4 a_{2} g_{1} g_{2}(\bmod 8) \equiv 4(\bmod 8) \\
& \quad \text { if } a_{2}+g_{1} \equiv 4 \quad(\bmod 8) .
\end{aligned}
$$

Calculating the 2-symbols of the forms $J\left(4 A_{4}\right)$, we get formulas (40).

The above arguments enable us to represent, up to equivalence over $\mathbb{Z}_{2}$, the first blocks $J\left(4 A_{4}\right)$ in the decomposition (38) as follows:

$$
\begin{aligned}
& J\left(4 A_{4}\right)=\left(\begin{array}{cc}
4 a_{1} & 1 \\
1 & \left(a_{1}+g_{1}\right) / 4
\end{array}\right) \oplus\left(\begin{array}{cc}
4 a_{2} & 1 \\
1 & \left(a_{2}+g_{2}\right) / 4
\end{array}\right) \quad \text { or } \\
& J\left(4 A_{4}\right)=\left(\begin{array}{cc}
4 a_{1} & 1 \\
1 & \left(a_{1}+g_{2}\right) / 4
\end{array}\right) \oplus\left(\begin{array}{cc}
4 a_{2} & 1 \\
1 & \left(a_{2}+g_{1}\right) / 4
\end{array}\right), \quad a_{1} \equiv a_{2}(\bmod 4) .
\end{aligned}
$$


TABLE 3

\begin{tabular}{|c|c|c|c|c|c|c|}
\hline$J\left(4 A_{4}\right)$ & $1_{\mathrm{I}, 0}^{+2}$ & $1_{\mathrm{I}, 4}^{-2}$ & $1_{\mathrm{I}, 2}^{+2}$ & $1_{\mathrm{I}, 6}^{-2}$ & $1_{\mathrm{I}, 6}^{+2}$ & $1_{\mathrm{I}, 2}^{-2}$ \\
\hline $4_{\mathrm{I}, 0}^{+2}$ & $1_{\mathrm{I}, 0}^{+4}, 1_{\mathrm{II}}^{+4}$ & $1_{\mathrm{I}, 4}^{-4}$ & & & & \\
\hline $4_{\mathrm{I}, 4}^{-2}$ & $1_{\mathrm{I}, 4}^{-4}$ & $1_{\mathrm{I}, 0}^{+4}, 1_{\mathrm{II}}^{+4}$ & & & & \\
\hline $4_{\mathrm{I}, 2}^{+2}$ & & & & & $1_{\mathrm{I}, 0}^{+4}, 1_{\mathrm{II}}^{+4}$ & $1_{\mathrm{I}, 4}^{-4}$ \\
\hline $4_{\mathrm{I}, 6}^{-2}$ & & & & & $1_{\mathrm{I}, 4}^{-4}$ & $1_{\mathrm{I}, 0}^{+4}, 1_{\mathrm{II}}^{+4}$ \\
\hline $4_{\mathrm{I}, 6}^{+2}$ & & & $1_{\mathrm{I}, 0}^{+4}, 1_{\mathrm{II}}^{+4}$ & $1_{\mathrm{I}, 4}^{-4}$ & & \\
\hline $4_{\mathrm{I}, 2}^{-2}$ & & & $1_{\mathrm{I}, 4}^{-4}$ & $1_{\mathrm{I}, 0}^{+4}, 1_{\mathrm{II}}^{+4}$ & & \\
\hline
\end{tabular}

Consequently, the forms $J\left(4 A_{4}\right)$ determine the minimal decomposable embeddings of the binary forms $A=4 A_{4}$ with odd $A_{4}$. The oddities of the constituents of $A=4 A_{4}$ are summed, and in both cases we have $\left|J\left(4 A_{4}\right)\right| \equiv\left|A_{4}\right| \cdot\left|G_{1}\right|(\bmod 8)$.

Table 3 is formed on the basis of formulas (39) and (40). The first row and the first column contain the 2-symbols of the binary forms $G_{1}$ and $A=4 A_{4}$, and the 2 -symbols of the forms $J\left(4 A_{4}\right)$ stand at the intersections of rows and columns.

Now we pass to forms $A=q A_{q}$ with an odd block $A_{q}$ of dimension $2, q=2^{\alpha}, \alpha \geq 3$. From (29) we obtain $G=G_{1} \oplus q G_{q}$, where $G_{1} \equiv-A_{q}(\bmod q)$. Therefore, $G_{1}$ is one of the forms given in (35), and we may assume that $G_{1}=-A_{q}$. In the congruence (5) we set $E=A_{q}$ and take $C=\left(C_{1} 0\right)$, where $C_{1}$ is a solution of the matrix congruence $A_{q}\left[C_{1}\right] \equiv-G_{1}(\bmod q)$. The group $S L_{2}\left(\mathbb{Z} / 2^{\alpha} \mathbb{Z}\right)$ contains $3 \cdot 2^{3 \alpha-2}$ elements.

First, let $\alpha=3$. The congruence $A_{q}\left[C_{1}\right] \equiv-G_{1}(\bmod q)$ is valid for the following matrices $C_{1}$ :

$$
\begin{aligned}
& \left(\begin{array}{cc} 
\pm 1 & 0 \\
0 & \pm 1
\end{array}\right),\left(\begin{array}{cc} 
\pm(q / 2-1) & 0 \\
0 & \pm(q / 2-1)
\end{array}\right),\left(\begin{array}{cc} 
\pm 1 & q / 2 \\
q / 2 & \pm 1
\end{array}\right) \\
& \left(\begin{array}{cc} 
\pm(q / 2-1) & q / 2 \\
q / 2 & \pm(q / 2-1)
\end{array}\right),\left(\begin{array}{cc}
q / 2 & \pm(q / 2-1) \\
\mp(q / 2-1) & q / 2
\end{array}\right) \\
& \left(\begin{array}{cc}
0 & \pm 1 \\
\mp 1 & 0
\end{array}\right),\left(\begin{array}{cc}
0 & \pm(q / 2-1) \\
\mp(q / 2-1) & 0
\end{array}\right),\left(\begin{array}{cc}
q / 2 & \pm 1 \\
\mp 1 & q / 2
\end{array}\right)
\end{aligned}
$$

these matrices form a group isomorphic to $C_{2} \times C_{2} \times C_{4}$. The solutions $C_{1}$ in (42) provide decompositions of the form $Q=J\left(q A_{q}\right) \oplus G_{q}$. For every matrix $C_{1}$ in (42), we describe the block $J\left(q A_{q}\right)$ up to equivalence over $\mathbb{Z}_{2}$ :

$$
\begin{aligned}
& J_{C_{1}^{(1)}}=\left(\begin{array}{cc}
q a_{1} & \pm 1 \\
\pm 1 & 0
\end{array}\right) \oplus\left(\begin{array}{cc}
q a_{2} & \pm 1 \\
\pm 1 & 0
\end{array}\right)=J_{\mathrm{II}} \oplus J_{\mathrm{II}}, \\
& J_{C_{1}^{(2)}}=\left(\begin{array}{cc}
q a_{1} & \pm(q / 2-1) \\
\pm(q / 2-1) & a_{1}\left(\frac{q}{4}-1\right)
\end{array}\right) \oplus\left(\begin{array}{cc}
q a_{2} & \pm(q / 2-1) \\
\pm(q / 2-1) & a_{2}\left(\frac{q}{4}-1\right)
\end{array}\right)=J_{\mathrm{I}} \oplus J_{\mathrm{I}}, \\
& J_{C_{1}^{(3)}}=\left(\begin{array}{cc}
q a_{1} & \pm 1 \\
\pm 1 & \frac{q}{4} a_{2}
\end{array}\right) \oplus\left(\begin{array}{cc}
q a_{2} & \pm 1 \\
\pm 1 & \frac{q}{4} a_{1}
\end{array}\right)=J_{\mathrm{II}} \oplus J_{\mathrm{II}}, \\
& J_{C_{1}^{(4)}}=\left(\begin{array}{cc}
q a_{1} & \pm(q / 2-1) \\
\pm(q / 2-1) & \frac{q}{4}\left(a_{1}+a_{2}\right)-a_{1}
\end{array}\right) \oplus\left(\begin{array}{cc}
q a_{2} & \pm(q / 2-1) \\
\pm(q / 2-1) & \frac{q}{4}\left(a_{1}+a_{2}\right)-a_{2}
\end{array}\right)=J_{\mathrm{I}} \oplus J_{\mathrm{I}} .
\end{aligned}
$$


In the case where $a_{1}=a_{2}$, we have

$$
\begin{aligned}
& J_{C_{1}^{(6)}}=\left(\begin{array}{cc}
q a_{1} & \pm 1 \\
\pm 1 & 0
\end{array}\right) \oplus\left(\begin{array}{cc}
q a_{2} & \mp 1 \\
\mp 1 & 0
\end{array}\right)=J_{\mathrm{II}} \oplus J_{\mathrm{II}}, \\
& J_{C_{1}^{(7)}}=\left(\begin{array}{cc}
q a_{1} & \pm(q / 2-1) \\
\pm(q / 2-1) & a_{1}\left(\frac{q}{4}-1\right)
\end{array}\right) \oplus\left(\begin{array}{cc}
q a_{2} & \mp(q / 2-1) \\
\mp(q / 2-1) & a_{2}\left(\frac{q}{4}-1\right)
\end{array}\right)=J_{\mathrm{I}} \oplus J_{\mathrm{I}}, \\
& J_{C_{1}^{(8)}}=\left(\begin{array}{cc}
q a_{1} & \pm 1 \\
\pm 1 & a_{2} \frac{q}{4}
\end{array}\right) \oplus\left(\begin{array}{cc}
q a_{2} & \mp 1 \\
\mp 1 & a_{1} \frac{q}{4}
\end{array}\right)=J_{\mathrm{II}} \oplus J_{\mathrm{II}}, \\
& J_{C_{1}^{(5)}}=\left(\begin{array}{cc}
q a_{1} & \pm(q / 2-1) \\
\pm(q / 2-1) & \frac{q}{4}\left(a_{1}+a_{2}\right)-a_{1}
\end{array}\right) \oplus\left(\begin{array}{cc}
q a_{2} & \mp(q / 2-1) \\
\mp(q / 2-1) & \frac{q}{4}\left(a_{1}+a_{2}\right)-a_{2}
\end{array}\right)=J_{\mathrm{I}} \oplus J_{\mathrm{I}} .
\end{aligned}
$$

In all cases, $\left|J_{i}\right| \equiv-1(\bmod 8)$ and $t\left(J_{i}\right) \equiv 0(\bmod 8), i=\mathrm{I}$, II.

In the general case where $\alpha \geq 3$, the solutions of the congruence $A_{q}\left[C_{1}\right] \equiv A_{q}(\bmod q)$ form the group

$$
\Gamma_{0}=\left\{\left(\begin{array}{cc}
c & c_{2} \\
c_{3} & c
\end{array}\right), \text { where } c_{3}^{2} a_{2} \equiv a_{1}\left(1-c^{2}\right)(\bmod q), c_{2} a_{1} \equiv-c_{3} a_{2}(\bmod q)\right\} .
$$

For each matrix $C_{1}$ in the group $\Gamma_{0}$, the block $J\left(q A_{q}\right)$ takes the form

$$
J\left(q A_{q}\right)=\left(\begin{array}{cccc}
q a_{1} & 0 & c & c_{2} \\
0 & q a_{2} & c_{3} & c \\
c & c_{3} & b_{1} & b_{2} \\
c_{2} & c & b_{2} & b_{3}
\end{array}\right) .
$$

where, $b_{1}=\frac{c^{2} a_{1}+c_{3}^{2} a_{2}-a_{1}}{q}, b_{2}=\frac{c c_{2} a_{1}+c c_{3} a_{2}}{q}$, and $b_{3}=\frac{c^{2} a_{2}+c_{2}^{2} a_{1}-a_{2}}{q}$. The form $J\left(q A_{q}\right)$ over $\mathbb{Z}_{2}$ is equivalent to

$$
\begin{aligned}
& \left(\begin{array}{cc}
q a_{1} & c \\
c & b_{1}
\end{array}\right) \oplus\left(\begin{array}{cc}
q a_{2} & c \\
c & b_{3}
\end{array}\right) \quad \text { for } \quad c \equiv 1 \quad(\bmod 2), \quad c_{2}, c_{3} \equiv 0 \quad(\bmod 2) \\
& \left(\begin{array}{cc}
q a_{1} & c_{2} \\
c_{2} & b_{3}
\end{array}\right) \oplus\left(\begin{array}{cc}
q a_{2} & c_{3} \\
c_{3} & b_{1}
\end{array}\right) \quad \text { for } c \equiv 0 \quad(\bmod 2), \quad c_{2}, c_{3} \equiv 1 \quad(\bmod 2)
\end{aligned}
$$

and the forms $J\left(q A_{q}\right)$ determine minimal decomposable embeddings of the binary forms $A=q A_{q}, q=2^{\alpha}, \alpha \geq 3$, with an odd block $A_{q}$. Calculations show that, depending on the evenness/oddness of the blocks $b_{1}$ and $b_{3}$, the form $J\left(q A_{q}\right)$ may have the 2-symbol $1_{\text {II }}^{+4}$ or $1_{I, 0}^{+4}$.

In Table 4, the first row and the first column contain the 2-symbols of the forms $G_{1}=-A_{q}$ and $A=q A_{q}, q=2^{\alpha}, \alpha \geq 3$. At the intersections of rows and columns, the corresponding forms $J\left(q A_{q}\right)$ are given.

For the general forms $A=A_{1} \oplus q A_{q}, \operatorname{dim} A_{q}=2$, with an odd block $A_{q}, q=2^{\alpha}$, $\alpha \geq 1$, formula (26) and the decomposition $Q=J\left(q A_{q}\right) \oplus G_{q}$ imply the formula

$$
G=G_{1} \oplus q(Q \ominus J(A)),
$$

where $J(A)=A_{1} \oplus J\left(q A_{q}\right)$ and the 2-symbols of minimal decomposable embeddings $J\left(q A_{q}\right)$ are calculated by formulas (36), (41), and (43), respectively; for $\alpha \geq 2$ they are given in Tables 3 and 4 , respectively.

4.4. The forms $A=q A_{q}$ with an even block $A_{q}$ of dimension $2, q=2^{\alpha}, \alpha \geq 1$, have a specific behavior over $\mathbb{Z}_{2}$. The local classification over $\mathbb{Z}_{2}$ implies that $A_{q}$ is one of the following two indecomposable forms:

$$
0_{\mathrm{II}}^{+}=\left(\begin{array}{ll}
0 & 1 \\
1 & 0
\end{array}\right), \quad 0_{\mathrm{II}}^{-}=\left(\begin{array}{ll}
2 & 1 \\
1 & 2
\end{array}\right)
$$


TABLE 4

\begin{tabular}{|c|c|c|c|c|c|c|}
\hline$J\left(q A_{q}\right)$ & $1_{\mathrm{I}, 0}^{+2}$ & $1_{\mathrm{I}, 4}^{-2}$ & $1_{\mathrm{I}, 2}^{+2}$ & $1_{\mathrm{I}, 6}^{-2}$ & $1_{\mathrm{I}, 6}^{+2}$ & $1_{\mathrm{I}, 2}^{-2}$ \\
\hline$q_{\mathrm{I}, 0}^{+2}$ & $1_{\mathrm{I}, 0}^{+4}, 1_{\mathrm{II}}^{+4}$ & & & & & \\
\hline$q_{\mathrm{I}, 4}^{-2}$ & & $1_{\mathrm{I}, 0}^{+4}, 1_{\mathrm{II}}^{+4}$ & & & & \\
\hline$q_{\mathrm{I}, 2}^{+2}$ & & & & & $1_{\mathrm{I}, 0}^{+4}, 1_{\mathrm{II}}^{+4}$ & \\
\hline$q_{\mathrm{I}, 6}^{-2}$ & & & & & & $1_{\mathrm{I}, 0}^{+4}, 1_{\mathrm{II}}^{+4}$ \\
\hline$q_{\mathrm{I}, 6}^{+2}$ & & & $1_{\mathrm{I}, 0}^{+4}, 1_{\mathrm{II}}^{+4}$ & & & \\
\hline$q_{\mathrm{I}, 2}^{-2}$ & & & & $1_{\mathrm{I}, 0}^{+4}, 1_{\mathrm{II}}^{+4}$ & & \\
\hline
\end{tabular}

The signs + and - denote the sign of the determinant, the number of the form indicates its oddity $t$, and the sign II shows that the form is even.

The case of $q=2$ was studied by Zhuravlev in 25], where automorphisms of the forms $G_{1}$ dual to $A_{2}$ were considered. Since $E=A_{2}^{-1} \equiv A_{2}(\bmod 2), G=G_{1} \oplus 2 G_{2}$, and, by (29), $G_{1} \equiv-E(\bmod 2)$, it follows that the forms $A_{2}$ and $G_{1}$ are $\mathbb{Z}_{2}$-equivalent to one of the forms in (45). Taking $C=\left(C_{1} 0\right)$ in (26), where $C_{1}$ is viewed modulo 2, we deduce that the congruence

$$
E\left[C_{1}\right] \equiv-G_{1} \quad(\bmod 2)
$$

is equivalent to the congruence $0_{\mathrm{II}}^{-}\left[C_{1}\right] \equiv 0_{\mathrm{II}}^{-}(\bmod 2)$. Its solutions constitute the group $S L_{2}(\mathbb{Z} / 2 \mathbb{Z})=O_{\mathbb{Z}}\left(0_{\text {II }}^{-}\right) / \bmod 2$, where $O_{\mathbb{Z}}\left(0_{\text {II }}^{-}\right)=$Aut $A_{2}$ is the automorphism group of the hexagonal lattice. The $\mathbb{Z}_{2}$-equivalence class of the form $Q$ as in (26) for $C_{1}$ from (48) depends on the coset of $C_{1} \cdot O_{\mathbb{Z}}\left(G_{1}\right) / \bmod 2$ (see [20]). From the matrix representation it follows that $O_{\mathbb{Z}}\left(0_{\mathrm{II}}^{+}\right)$is a subgroup in $O_{\mathbb{Z}}\left(0_{\mathrm{II}}^{-}\right)$, but not a normal subgroup, and $O_{\mathbb{Z}}\left(0_{\mathrm{II}}^{-}\right) / O_{\mathbb{Z}}\left(0_{\mathrm{II}}^{+}\right)=\left\{1, \sigma, \sigma^{2}\right\}$ is the set of its cosets, $\sigma=\left(\begin{array}{cc}0 & -1 \\ 1 & 1\end{array}\right)$. Thus, to calculate the invariants of the form $Q$ in (26), we can restrict ourselves in the congruence (46) to the solutions $C_{1}=\left(\begin{array}{ll}1 & 0 \\ 0 & 1\end{array}\right)$ for $G_{1}=0_{\mathrm{II}}^{-}$and $C_{1}=\left(\begin{array}{ll}1 & 0 \\ 0 & 1\end{array}\right),\left(\begin{array}{ll}0 & 1 \\ 1 & 1\end{array}\right),\left(\begin{array}{ll}1 & 1 \\ 1 & 0\end{array}\right)$ for $G_{1}=0_{\mathrm{II}}^{+}$, respectively.

Using the said above and formula (26), in 25) Zhuravlev obtained all distinct minimal indecomposable embeddings $J\left(2 A_{2}\right)$ of binary forms $2 A_{2}$ with an even block $A_{2}$ over $\mathbb{Z}_{2}$ and computed their 2-symbols:

$$
2_{J\left(2 A_{2}\right)}= \begin{cases}1_{\mathrm{II}}^{+4}, 1_{\mathrm{I}, 0}^{+4} & \text { for } 2_{2 A_{2}}=2_{\mathrm{II}}^{+2} \text { and } 2_{G_{1}}=1_{\mathrm{II}}^{+2}, \\ 1_{\mathrm{II}}^{+4} & \text { for } 2_{2 A_{2}}=2_{\mathrm{II}}^{-2} \text { and } 2_{G_{1}}=1_{\mathrm{II}}^{-2}, \\ 1_{\mathrm{I}, 4}^{-4} & \text { for } 2_{2 A_{2}}=2_{\mathrm{II}}^{\beta_{1} 2} \text { and } 2_{G_{1}}=1_{\mathrm{II}}^{\beta_{2} 2}, \quad \beta_{1} \beta_{2}=-1, \quad \beta_{i}= \pm 1 .\end{cases}
$$

Now we pass to the next case of forms $A=q A_{q}$, where $q=2^{\alpha}, \alpha \geq 2, \operatorname{dim} A_{q}=2$, and the block $A_{q}$ is even. From (29) we see that $G=G_{1} \oplus q G_{q}$, where $G_{1} \equiv-E$ $(\bmod q)$. Since $E=A_{q}^{-1} \equiv A_{q}(\bmod q)$, the forms $A_{q}$ and $G_{1}$ are equivalent to one of the forms (45) over $\mathbb{Z}_{2}$. In formula (26) we set $E=A_{q}$ and $C=\left(C_{1} 0\right)$, where the $(2 \times 2)$-matrix $C_{1}$ is a solution of the matrix congruence

$$
A_{q}\left[C_{1}\right] \equiv-G_{1} \quad(\bmod q),
$$

which splits into two congruences $0_{\mathrm{II}}^{+}\left[C_{1}\right] \equiv 0_{\mathrm{II}}^{+}(\bmod q)$ and $0_{\mathrm{II}}^{-}\left[C_{1}\right] \equiv 0_{\mathrm{II}}^{-}(\bmod q)$. This implies that the block $C_{1}$ belongs to the group $O\left(0_{\mathrm{II}}^{+} / \bmod q\right)$ or to $O\left(0_{\mathrm{II}}^{-} / \bmod q\right)$, which are orthogonal groups over the ring of residues $\mathbb{Z}_{p} / q \mathbb{Z}_{p}$. 
First, we consider the congruence $0_{\mathrm{II}}^{+}\left[C_{1}\right] \equiv 0_{\mathrm{II}}^{+}(\bmod q), q \geq 4$. Its solutions form the group (a subgroup of $S L_{2}(\mathbb{Z} / q \mathbb{Z})$ )

$$
\Gamma_{1}=\left\{\left(\begin{array}{cc}
c & 0 \\
0 & c^{-1}
\end{array}\right),\left(\begin{array}{cc}
c & q / 2 \\
q / 2 & c^{-1}
\end{array}\right),\left(\begin{array}{cc}
c & 0 \\
q / 2 & c^{-1}
\end{array}\right),\left(\begin{array}{cc}
c & q / 2 \\
0 & c^{-1}
\end{array}\right)\right\}
$$

where $c= \pm 1, \pm 3, \ldots, \pm(q / 2-1)$ are odd numbers and $c c^{-1} \equiv 1(\bmod q)$. The order of the group $\Gamma_{1}$ is equal to $2 q$, and $\Gamma_{1}$ is isomorphic to the group $C_{2} \times C_{2} \times C_{2} \times C_{2^{\alpha-2}}$, where $q=2^{\alpha}, \alpha \geq 2$. For such $C_{1}$, formula (26) yields the decomposition

$$
Q=J\left(q A_{q}\right) \oplus G_{q}
$$

with the embedding $J\left(q A_{q}\right)=\left(\begin{array}{cc}q A_{q} & C_{1} \\ { }^{t} C_{1} & Q^{\prime \prime}\end{array}\right), Q^{\prime \prime}=\left(A_{q}\left[C_{1}\right]+G_{1}\right) / q$, minimal for the form $q A_{q}$.

If $q=4$, the group $\Gamma_{1}$ contains eight matrices $C_{1}$ :

$$
\left(\begin{array}{cc} 
\pm 1 & 0 \\
0 & \pm 1
\end{array}\right),\left(\begin{array}{cc} 
\pm 1 & 2 \\
2 & \pm 1
\end{array}\right),\left(\begin{array}{cc} 
\pm 1 & 2 \\
0 & \pm 1
\end{array}\right),\left(\begin{array}{cc} 
\pm 1 & 0 \\
2 & \pm 1
\end{array}\right)
$$

and is isomorphic to the group $C_{2} \times C_{2} \times C_{2}$. To compute the blocks $Q^{\prime \prime}$ modulo 8, we take the following matrices $G_{1}$ :

$$
G_{1}^{(1)}=\left(\begin{array}{cc}
0 & -1 \\
-1 & 0
\end{array}\right), \quad G_{1}^{(2)}=\left(\begin{array}{cc}
4 & -1 \\
-1 & 4
\end{array}\right), \quad G_{1}^{(3)}=\left(\begin{array}{cc}
4 & -1 \\
-1 & 0
\end{array}\right),
$$

where $G_{1}^{(1)} \equiv G_{1}^{(2)} \equiv G_{1}^{(3)} \equiv-0_{\mathrm{II}}^{+}(\bmod 4)$ and $2_{G_{1}^{(i)}}=1_{\mathrm{II}}^{+2}, i=1,2,3$. This choice guarantees the presence of two parity types of the block $Q^{\prime \prime}$ for every matrix $C_{1}$ and, respectively, yields two types of the forms $J\left(4 A_{4}\right)$, nonequivalent over $\mathbb{Z}_{2}$ :

$$
\begin{aligned}
& J_{C_{1}^{(1)}}^{G_{1}^{(1)}}=\left(\begin{array}{cccc}
0 & 4 & \pm 1 & 0 \\
4 & 0 & 0 & \pm 1 \\
\pm 1 & 0 & 0 & 0 \\
0 & \pm 1 & 0 & 0
\end{array}\right) \sim\left(\left(\begin{array}{cc}
0 & \pm 1 \\
\pm 1 & 0
\end{array}\right) \oplus\left(\begin{array}{cc}
0 & \pm 1 \\
\pm 1 & 0
\end{array}\right)=J_{\mathrm{II}} \oplus J_{\mathrm{II}},\right. \\
& J_{C_{1}^{(1)}}^{G_{1}^{(2)}}=\left(\begin{array}{cccc}
0 & 4 & \pm 1 & 0 \\
4 & 0 & 0 & \pm 1 \\
\pm 1 & 0 & 1 & 0 \\
0 & \pm 1 & 0 & 1
\end{array}\right) \sim\left(\begin{array}{cc}
0 & \pm 1 \\
\pm 1 & 1
\end{array}\right) \oplus\left(\begin{array}{cc}
0 & \pm 1 \\
\pm 1 & 1
\end{array}\right)=J_{\mathrm{I}} \oplus J_{\mathrm{I}} \\
& J_{C_{1}^{(2)}}^{G^{(1)}}=\left(\begin{array}{cccc}
0 & 4 & \pm 1 & 2 \\
4 & 0 & 2 & \pm 1 \\
\pm 1 & 2 & \pm 1 & 1 \\
2 & \pm 1 & 1 & \pm 1
\end{array}\right) \sim\left(\begin{array}{cc}
0 & \pm 1 \\
\pm 1 & \pm 1
\end{array}\right) \oplus\left(\begin{array}{cc}
0 & \pm 1 \\
\pm 1 & \pm 1
\end{array}\right)=J_{\mathrm{I}} \oplus J_{\mathrm{I}} \\
& J_{C_{1}^{(2)}}^{G^{(2)}}=\left(\begin{array}{cccc}
0 & 4 & \pm 1 & 2 \\
4 & 0 & 2 & \pm 1 \\
\pm 1 & 2 & 1 \pm 1 & 1 \\
2 & \pm 1 & 1 & 1 \pm 1
\end{array}\right) \sim\left(\begin{array}{cc}
0 & \pm 1 \\
\pm 1 & 1 \pm 1
\end{array}\right) \oplus\left(\begin{array}{cc}
0 & \pm 1 \\
\pm 1 & -3 \pm 1
\end{array}\right)=J_{\mathrm{II}} \oplus J_{\mathrm{II}} \\
& J_{C_{1}^{(3)}}^{G_{1}^{(1)}}=\left(\begin{array}{cccc}
0 & 4 & \pm 1 & 2 \\
4 & 0 & 0 & \pm 1 \\
\pm 1 & 0 & 0 & 0 \\
2 & \pm 1 & 0 & \pm 1
\end{array}\right) \sim\left(\begin{array}{cc}
0 & \pm 1 \\
\pm 1 & 0
\end{array}\right) \oplus\left(\begin{array}{cc}
0 & \pm 1 \\
\pm 1 & \pm 1
\end{array}\right)=J_{\mathrm{II}} \oplus J_{\mathrm{I}},
\end{aligned}
$$




$$
\begin{aligned}
& J_{C_{1}^{(3)}}^{G_{1}^{(3)}}=\left(\begin{array}{cccc}
0 & 4 & \pm 1 & 2 \\
4 & 0 & 0 & \pm 1 \\
\pm 1 & 0 & 0 & 0 \\
2 & \pm 1 & 0 & 1 \pm 1
\end{array}\right) \sim\left(\begin{array}{cc}
0 & \pm 1 \\
\pm 1 & 0
\end{array}\right) \oplus\left(\begin{array}{cc}
0 & \pm 1 \\
\pm 1 & 1 \pm 1
\end{array}\right)=J_{\mathrm{II}} \oplus J_{\mathrm{II}}, \\
& J_{C_{1}^{(4)}}^{G^{(1)}}=\left(\begin{array}{cccc}
0 & 4 & \pm 1 & 0 \\
4 & 0 & 2 & \pm 1 \\
\pm 1 & 2 & \pm 1 & 0 \\
0 & \pm 1 & 0 & 0
\end{array}\right) \sim\left(\begin{array}{cc}
0 & \pm 1 \\
\pm 1 & 0
\end{array}\right) \oplus\left(\begin{array}{cc}
0 & \pm 1 \\
\pm 1 & \pm 1
\end{array}\right)=J_{\mathrm{II}} \oplus J_{\mathrm{I}}, \\
& J_{C_{1}^{(4)}}^{G^{(3)}}=\left(\begin{array}{cccc}
0 & 4 & \pm 1 & 0 \\
4 & 0 & 2 & \pm 1 \\
\pm 1 & 2 & 1 \pm 1 & 0 \\
0 & \pm 1 & 0 & 0
\end{array}\right) \sim\left(\begin{array}{cc}
0 & \pm 1 \\
\pm 1 & 0
\end{array}\right) \oplus\left(\begin{array}{cc}
0 & \pm 1 \\
\pm 1 & 1 \pm 1
\end{array}\right)=J_{\mathrm{II}} \oplus J_{\mathrm{II}},
\end{aligned}
$$

with the 2 -symbols $1_{\mathrm{I}, 0}^{+4}$ and $1_{\mathrm{II}}^{+4}$. The above forms $J\left(4 A_{4}\right)$ determine the minimal indecomposable embeddings of the binary forms $A=4 A_{4}, A_{4}=0_{\mathrm{II}}^{+2}$, over $\mathbb{Z}_{2}$.

Considering the cases where $q \geq 8$, we may assume that $G_{1}=-A_{q}$. By formula (49), for the forms $A_{q}=0_{\mathrm{II}}^{+}$the matrices $C_{1}$ provide the following forms $J\left(q A_{q}\right)$ of dimension 4 :

$$
\begin{aligned}
J_{C_{1}^{(1)}} & =\left(\begin{array}{cccc}
0 & q & c & 0 \\
q & 0 & 0 & c^{-1} \\
c & 0 & 0 & 0 \\
0 & c^{-1} & 0 & 0
\end{array}\right) \sim\left(\begin{array}{cc}
0 & c \\
c & 0
\end{array}\right) \oplus\left(\begin{array}{cc}
0 & c^{-1} \\
c^{-1} & 0
\end{array}\right)=J_{\mathrm{II}} \oplus J_{\mathrm{II}}, \\
J_{C_{1}^{(2)}} & =\left(\begin{array}{cccc}
0 & q & c & q / 2 \\
q & 0 & q / 2 & c^{-1} \\
c & q / 2 & c & q / 4 \\
q / 2 & c^{-1} & q / 4 & c^{-1}
\end{array}\right) \sim\left(\begin{array}{ll}
0 & c \\
c & c
\end{array}\right) \oplus\left(\begin{array}{cc}
0 & c^{-1} \\
c^{-1} & c^{-1}
\end{array}\right)=J_{\mathrm{I}} \oplus J_{\mathrm{I}}, \\
J_{C_{1}^{(3)}} & =\left(\begin{array}{cccc}
0 & q & c & 0 \\
q & 0 & q / 2 & c^{-1} \\
c & q / 2 & c & 0 \\
0 & c^{-1} & 0 & 0
\end{array}\right) \sim\left(\begin{array}{ll}
0 & c \\
c & c
\end{array}\right) \oplus\left(\begin{array}{cc}
0 & c^{-1} \\
c^{-1} & 0
\end{array}\right)=J_{\mathrm{I}} \oplus J_{\mathrm{II}}, \\
J_{C_{1}^{(4)}} & =\left(\begin{array}{cccc}
0 & q & c & q / 2 \\
q & 0 & 0 & c^{-1} \\
c & 0 & 0 & 0 \\
q / 2 & c^{-1} & 0 & c^{-1}
\end{array}\right) \sim\left(\begin{array}{ll}
0 & c \\
c & 0
\end{array}\right) \oplus\left(\begin{array}{cc}
0 & c^{-1} \\
c^{-1} & c^{-1}
\end{array}\right)=J_{\mathrm{II}} \oplus J_{\mathrm{I}},
\end{aligned}
$$

with the 2-symbols $2_{J_{C_{1}^{(1)}}}=1_{\mathrm{II}}^{+4}$ and $2_{J_{C_{1}^{(2)}}}=2_{J_{C_{1}^{(3)}}}=2_{J_{C_{1}^{(4)}}}=1_{\mathrm{I}, 0}^{+4}$. Consequently, the $J\left(q A_{q}\right)$ determine all distinct minimal indecomposable embeddings of the binary forms $A=q A_{q}, A_{q}=0_{\mathrm{II}}^{+2}, q=2^{\alpha}, \alpha \geq 3$, over $\mathbb{Z}_{2}$.

Next, we consider the congruence $0_{\mathrm{II}}^{-}\left[C_{1}\right] \equiv 0_{\mathrm{II}}^{-}(\bmod q), q=2^{\alpha}, \alpha \geq 2$; its solutions constitute the following group (a subgroup of $S L_{2}(\mathbb{Z} / q \mathbb{Z})$ ):

$$
\Gamma_{2}=\left\{\Gamma_{3} \cup \sigma \Gamma_{3} \cup \sigma^{2} \Gamma_{3}\right\},
$$

where

$$
\left.\Gamma_{3}=\left\{\begin{array}{cc}
c & x z \\
y z & c^{-1}
\end{array}\right), \begin{array}{l}
c^{2}+c^{-2} \equiv 2\left(\bmod \frac{q}{2}\right), z x \equiv c-c^{-1}\left(\bmod \frac{q}{2}\right) \\
x \equiv-y\left(\bmod \frac{q}{2 z}\right)
\end{array}\right\}
$$




\section{TABLE 5}

\begin{tabular}{|c|c|c|}
\hline$J\left(q A_{q}\right)$ & $1_{\mathrm{II}}^{+2}$ & $1_{\mathrm{II}}^{-2}$ \\
\hline$q_{\mathrm{II}}^{+2}$ & $1_{\mathrm{II}}^{+4}, 1_{\mathrm{I}, 0}^{+4}$ & \\
\hline$q_{\mathrm{II}}^{-2}$ & & $1_{\mathrm{II}}^{+4}, 1_{\mathrm{I}, 0}^{+4}$ \\
\hline
\end{tabular}

Here $c \in\{ \pm 1, \pm 3, \ldots, \pm(q / 2-1)\}$ and $c c^{-1} \equiv 1(\bmod q)$. For $q=2^{\alpha}$, we define

$$
z= \begin{cases}2^{t} & \text { if } \alpha=2 t \\ 2^{t+1} & \text { if } \alpha=2 t+1 .\end{cases}
$$

Similarly, formula (26) for $C_{1} \in \Gamma_{2}$ yields the decomposition (49) with the embedding $J\left(q A_{q}\right)=\left(\begin{array}{cc}q A_{q} & C_{1} \\ { }^{t} C_{1} & Q^{\prime \prime}\end{array}\right)$ minimal for the form $q A_{q}$, where the block $Q^{\prime \prime}$ is equal to $\left(A_{q}\left[C_{1}\right]+\right.$ $\left.G_{1}\right) / q$.

For $q=4$, the group $\Gamma_{2}$ contains the following 24 matrices $C_{1}$ (including (50)):

$$
\begin{aligned}
& \left(\begin{array}{cc}
0 & \pm 1 \\
\mp 1 & \pm 1
\end{array}\right),\left(\begin{array}{cc}
0 & \mp 1 \\
\pm 1 & \pm 1
\end{array}\right),\left(\begin{array}{cc} 
\pm 1 & \mp 1 \\
\pm 1 & 0
\end{array}\right),\left(\begin{array}{cc} 
\pm 1 & \pm 1 \\
\mp 1 & 0
\end{array}\right) \\
& \left(\begin{array}{cc}
1 & \pm 1 \\
\pm 1 & 2
\end{array}\right),\left(\begin{array}{cc}
2 & \pm 1 \\
\pm 1 & 1
\end{array}\right),\left(\begin{array}{cc}
-1 & \pm 1 \\
\pm 1 & 2
\end{array}\right),\left(\begin{array}{cc}
2 & \pm 1 \\
\pm 1 & -1
\end{array}\right)
\end{aligned}
$$

they constitute a group isomorphic to the group $C_{2} \times D_{6}$, where $D_{6}$ is the automorphism group of the hexagonal lattice, which is isomorphic to the dihedral group of symmetries of a regular hexagon.

To compute the blocks $Q^{\prime \prime}$ modulo 8 , we take the following matrices $G_{1}$ :

$$
\left(\begin{array}{cc}
2 & -1 \\
-1 & 2
\end{array}\right),\left(\begin{array}{cc}
6 & -1 \\
-1 & 6
\end{array}\right),\left(\begin{array}{cc}
2 & -1 \\
-1 & 6
\end{array}\right),\left(\begin{array}{cc}
6 & -1 \\
-1 & 2
\end{array}\right)
$$

where $G_{1}^{(1)} \equiv G_{1}^{(2)} \equiv G_{1}^{(3)} \equiv G_{1}^{(4)} \equiv-0_{\mathrm{II}}^{-}(\bmod 4)$ and $2_{G_{1}^{(i)}}=1_{\mathrm{II}}^{-2}, i=1,2,3$. This choice guarantees the presence of two parity types of the block $Q^{\prime \prime}$ for every matrix $C_{1}$ and provides two types of the forms $J\left(4 A_{4}\right)$, nonequivalent over $\mathbb{Z}_{2}$, with the 2 -symbols $1_{\mathrm{I}, 0}^{+4}$ and $1_{\mathrm{II}}^{+4}$, respectively (we omit calculations, which are similar to these applied for the congruence $\left.0_{\mathrm{II}}^{+2}\left[C_{1}\right] \equiv 0_{\mathrm{II}}^{+2}(\bmod 4)\right)$.

When treating the cases where $q \geq 8$, we may assume that $G_{1}=-A_{q}$. By formula (49), for the forms $A_{q}=0_{\mathrm{II}}^{-}$the matrices $C_{1}$ yield the forms $J\left(q A_{q}\right)$ of dimension 4 , which are equivalent over $\mathbb{Z}_{2}$ to the following direct sum of forms:

$$
J_{C_{1}} \sim J_{\sigma C_{1}} \sim J_{\sigma^{2} C_{1}} \sim\left(\begin{array}{cc}
2 q & c \\
c & m_{1}
\end{array}\right) \oplus\left(\begin{array}{cc}
2 q & c^{-1} \\
c^{-1} & m_{2}
\end{array}\right)
$$

where $m_{1}=\frac{2 c^{2}+2 c y z+2 y^{2} z^{2}-2}{q}$, and $m_{2}=\frac{2\left(c^{-1}\right)^{2}+2 x^{2} z^{2}+2 x z c^{-1}-2}{q}$, and $\operatorname{det} J_{1}, \operatorname{det} J_{2} \equiv-1$ $(\bmod 8) ;$ consequently, $\operatorname{det} J \equiv 1(\bmod 8)$. Therefore, the forms $J\left(q A_{q}\right)$ given in (52) determine all distinct minimal indecomposable embeddings of the binary forms $A=q A_{q}$, $A_{q}=0_{\mathrm{II}}^{-2}, q=2^{\alpha}, \alpha \geq 3$, over $\mathbb{Z}_{2}$. Since the numbers $m_{1}$ and $m_{2}$ may be of any parity, we see that the form $J\left(q A_{q}\right)$ may have the 2 -symbol $1_{\mathrm{I}, 0}^{+4}$ or $1_{\mathrm{II}}^{+4}$.

The above arguments allow us is to compose Table 5. The first row contains the 2 -symbols of the binary even forms $G_{1}$, the first column contains the 2-symbols of the binary forms $q A_{q}$ with an even block $A_{q}$, and the intersections of rows and columns are occupied by the 2 -symbols of the forms $J\left(q A_{q}\right), q=2^{\alpha}, \alpha \geq 2$. 
If the form $A=A_{1} \oplus q A_{q}$ has an even two-dimensional block $A_{q}$, then for the forms $G$ we still have formula (44) in which $G_{1}=0_{\mathrm{II}}^{ \pm 2}, J(A)=A_{1} \oplus J\left(q A_{q}\right)$, and the 2-symbols of the forms $J\left(q A_{q}\right)$ are computed by formulas (47) for $\alpha=1$ and are given in Table 5 for $\alpha \geq 2$.

4.5. Consider the representations of the forms

$$
A=q_{1} A_{q_{1}} \oplus q_{2} A_{q_{2}}, \quad \operatorname{dim} A_{q_{1}}=\operatorname{dim} A_{q_{2}}=1, \quad q_{2}>q_{1} \geq 2, q_{i}=2^{\alpha_{i}}, i=1,2,
$$

with level $a=q_{2}$, by forms $Q$ with odd determinant.

With the help of the minimal embeddings for one-dimensional forms $A=q A_{q}$ over the ring $\mathbb{Z}_{2}$ (see Subsection 4.2), we can find the local invariants of the forms $J(A)=$ $J\left(q_{1} A_{q_{1}}\right) \oplus J\left(q_{2} A_{q_{2}}\right), \operatorname{dim} A_{q_{1}}=\operatorname{dim} A_{q_{2}}=1$ by straightforward calculations:

$$
\begin{array}{ll}
1_{\mathrm{I}, 2}^{ \pm 4}, 1_{\mathrm{I}, 4}^{ \pm 4}, 1_{\mathrm{I}, 6}^{ \pm 4}, 1_{\mathrm{II}}^{ \pm 4} & \text { for } A=2 A_{2} \oplus 4 A_{4} ; \\
1_{\mathrm{I}, 0}^{ \pm 4}, 1_{\mathrm{II}}^{ \pm 4}, 1_{\mathrm{I}, 2}^{ \pm 4} & \text { for } A=2 A_{2} \oplus q_{2} A_{q_{2}}, q_{2} \geq 8,2_{2 A_{2}}=2_{I, 1}^{+1} \text { or } 2_{2 A_{2}}=2_{\mathrm{I}, 5}^{-1} ; \\
1_{\mathrm{I}, 0}^{ \pm 4}, 1_{\mathrm{II}}^{ \pm 4}, 1_{\mathrm{I}, 6}^{ \pm 4} & \text { for } A=2 A_{2} \oplus q_{2} A_{q_{2}}, q_{2} \geq 8,2_{2 A_{2}}=2_{I, 7}^{+1} \text { or } 2_{2 A_{2}}=2_{\mathrm{I}, 3}^{-1} ; \\
1_{\mathrm{I}, 0}^{+4}, 1_{\mathrm{I}, 4}^{-4}, 1_{\mathrm{II}}^{+4} & \text { for } A=4 A_{2} \oplus q_{2} A_{q_{2}}, q_{2} \geq 8 ; \\
1_{\mathrm{I}, 0}^{+4}, 1_{\mathrm{II}}^{+4} & \text { for } A=q_{1} A_{q_{1}} \oplus q_{2} A_{q_{2}}, q_{2}>q_{1} \geq 8 .
\end{array}
$$

\section{§5. Primitive 2-Universality for QUAdRatic Forms OF SPECial Kind}

5.1. The following result gives a complete answer to the question as to when a quadratic form $Q$ with $\operatorname{dim} Q \geq 5$ and odd determinant $|Q|$ is a primitively $2 \sim$ universal form.

Theorem 5. Let $Q$ be a positive definite, classically integral quadratic form of dimension at least 5 with odd determinant. If the 2 -symbol of the form $Q$ is equal to

$$
1_{\mathrm{I}, \pm 3}^{-5}, 1_{\mathrm{I}, \pm 1}^{+5}, 1_{\mathrm{I}, \pm 2}^{ \pm 6}, 1_{\mathrm{I}, 4}^{-6}, 1_{\mathrm{I}, 0}^{+6}, 1_{\mathrm{I}, t}^{ \pm n}(n \geq 7, t \equiv n \quad(\bmod 2))
$$

then $Q$ is a primitively $2 \sim$ universal form. If the 2 -symbol of the form $Q$ is equal to

$$
1_{\mathrm{I}, \pm 3}^{+5}, 1_{\mathrm{I}, \pm 1}^{-5}, 1_{\mathrm{I}, 4}^{+6}, 1_{\mathrm{I}, 0}^{-6}, 1_{\mathrm{II}}^{ \pm n}(n=2 t, n \geq 6),
$$

then $Q$ is not a primitively $2 \sim$ universal form.

Proof. We study the embeddings over $\mathbb{Z}_{2}$ of binary forms $A$ in forms $Q$ that have odd determinant and dimension at least 5. For this, we use the results of Subsections 4.2-4.5.

For every form $A=q A_{q}, q=2^{\alpha}, \alpha \geq 1$, and a block $G_{1}$, we find the minimal embeddings $J\left(q A_{q}\right)$. Next, we use the 2-symbols of the quaternary forms $J\left(q A_{q}\right)$ to calculate the 2-symbols of the forms $G=G_{1} \oplus q G_{q}$. The calculation of the 2-symbols of the forms $q G_{q}$ reduces to the following subtraction formulas for 2-symbols:

$$
\begin{aligned}
& 1_{\mathrm{I}, t_{1}}^{\epsilon_{1} n_{1}} \ominus 1_{\mathrm{II}}^{\epsilon_{2} n_{2}}=1_{I, t_{1}}^{\epsilon_{1} \epsilon_{2}\left(n_{1}-n_{2}\right)}, \quad 1_{\mathrm{II}}^{\epsilon_{1} n_{1}} \ominus 1_{\mathrm{II}}^{\epsilon_{2} n_{2}}=1_{\mathrm{II}}^{\epsilon_{1} \epsilon_{2}\left(n_{1}-n_{2}\right)}, \\
& 1_{\mathrm{I}, t_{1}}^{\epsilon_{1} n_{1}} \ominus 1_{\mathrm{I}, t_{2}}^{\epsilon_{2} n_{2}}=1_{\mathrm{I}, t_{1}-t_{2}}^{\epsilon_{1} \epsilon_{2}\left(n_{1}-n_{2}\right)} \text { or } 1_{\mathrm{II}}^{\epsilon_{1} \epsilon_{2}\left(n_{1}-n_{2}\right)} \text { if } t_{1} \equiv t_{2} \quad(\bmod 8) .
\end{aligned}
$$

The operation $\ominus$ is well defined only if the corresponding 2-symbol on the right exists (see [23]). If two forms $G$ and $G^{\prime}$ belong to one and the same equivalence class $\{G\}$ over $\mathbb{Z}_{2}$, then their 2 -symbols are equivalent $\left(2_{G} \sim 2_{G^{\prime}}\right)$ and are mapped one to the other by elementary transformations: fusion of oddities and signs walk along a train (see [23]). If we agree to pose a common sign at a certain place of the trains occurring in the 2 -symbols, then the bijection $G \sim_{2} G^{\prime} \Leftrightarrow 2_{G}^{c a n}=2_{G^{\prime}}^{c a n}$ is restored.

First, we consider quadratic forms $Q$ of dimension 5 with odd determinant. In this case, the 2-symbols of the forms $G$ can be written explicitly. We show the calculation of the 2-symbol of the form $G$ by the example of the embedding of the form $A=2 A_{2}, \operatorname{dim} A_{2}=2$, with the 2 -symbol $2_{A}=2_{I, 4}^{-2}$ in the form $Q$ with the 2 -symbol 
TABLE 6

\begin{tabular}{|c|c|c|c|c|c|c|c|c|}
\hline $2_{G}$ & $1_{\mathrm{I}, 7}^{+5}$ & $1_{\mathrm{I}, 7}^{-5}$ & $1_{\mathrm{I}, 3}^{-5}$ & $1_{\mathrm{I}, 5}^{+5}$ & $1_{\mathrm{I}, 5}^{-5}$ & $1_{\mathrm{I}, 1}^{+5}$ & $1_{\mathrm{I}, 1}^{-5}$ & $1_{\mathrm{I}, 3}^{+5}$ \\
\hline $2_{\mathrm{I}, 0}^{+2}$ & {$\left[1^{2} 2^{1}\right]_{7}$} & {$\left[1^{2} 2^{-1}\right]_{7}$} & {$\left[1^{2} 2^{-1}\right]_{3}$} & {$\left[1^{2} 2^{1}\right]_{5}$} & {$\left[1^{2} 2^{-1}\right]_{5}$} & {$\left[1^{2} 2^{1}\right]_{1}$} & {$\left[1^{2} 2^{-1}\right]_{1}$} & {$\left[1^{2} 2^{1}\right]_{3}$} \\
\hline $2_{\mathrm{I}, 4}^{-2}$ & {$\left[1^{2} 2^{-1}\right]_{7}$} & {$\left[1^{2} 2^{1}\right]_{7}$} & {$\left[1^{2} 2^{1}\right]_{3}$} & {$\left[1^{2} 2^{-1}\right]_{5}$} & {$\left[1^{2} 2^{1}\right]_{5}$} & {$\left[1^{2} 2^{-1}\right]_{1}$} & {$\left[1^{2} 2^{1}\right]_{1}$} & {$\left[1^{2} 2^{-1}\right]_{3}$} \\
\hline $2_{\mathrm{I}, 2}^{+2}$ & {$\left[1^{2} 2^{1}\right]_{5}$} & {$\left[1^{2} 2^{-1}\right]_{5}$} & {$\left[1^{2} 2^{-1}\right]_{1}$} & {$\left[1^{2} 2^{1}\right]_{3}$} & {$\left[1^{2} 2^{-1}\right]_{3}$} & {$\left[1^{2} 2^{1}\right]_{7}$} & {$\left[1^{2} 2^{-1}\right]_{7}$} & {$\left[1^{2} 2^{1}\right]_{1}$} \\
\hline $2_{\mathrm{I}, 6}^{-2}$ & {$\left[1^{2} 2^{-1}\right]_{5}$} & {$\left[1^{2} 2^{1}\right]_{5}$} & {$\left[1^{2} 2^{1}\right]_{1}$} & {$\left[1^{2} 2^{-1}\right]_{3}$} & {$\left[1^{2} 2^{1}\right]_{3}$} & {$\left[1^{2} 2^{-1}\right]_{7}$} & {$\left[1^{2} 2^{1}\right]_{7}$} & {$\left[1^{2} 2^{-1}\right]_{1}$} \\
\hline $2_{\mathrm{I}, 6}^{+2}$ & {$\left[1^{2} 2^{1}\right]_{1}$} & {$\left[1^{2} 2^{-1}\right]_{1}$} & {$\left[1^{2} 2^{-1}\right]_{5}$} & {$\left[1^{2} 2^{1}\right]_{7}$} & {$\left[1^{2} 2^{-1}\right]_{7}$} & {$\left[1^{2} 2^{1}\right]_{3}$} & {$\left[1^{2} 2^{-1}\right]_{3}$} & {$\left[1^{2} 2^{1}\right]_{5}$} \\
\hline $2_{\mathrm{I}, 2}^{-2}$ & {$\left[1^{2} 2^{-1}\right]_{1}$} & {$\left[1^{2} 2^{1}\right]_{1}$} & {$\left[1^{2} 2^{1}\right]_{5}$} & {$\left[1^{2} 2^{-1}\right]_{7}$} & {$\left[1^{2} 2^{1}\right]_{7}$} & {$\left[1^{2} 2^{-1}\right]_{3}$} & {$\left[1^{2} 2^{1}\right]_{3}$} & {$\left[1^{2} 2^{-1}\right]_{5}$} \\
\hline $2_{\mathrm{II}}^{+2}$ & $1_{\mathrm{II}}^{+2}\left[2^{1}\right]_{7}$ & & $1_{\mathrm{II}}^{+2}\left[2^{-1}\right]_{3}$ & & $1_{\mathrm{II}}^{+2}\left[2^{-1}\right]_{5}$ & $1_{\mathrm{II}}^{+2}\left[2^{1}\right]_{1}$ & & \\
\hline $2_{\mathrm{II}}^{-2}$ & $1_{\mathrm{II}}^{+2}\left[2^{-1}\right]_{3}$ & & $1_{\mathrm{II}}^{+2}\left[2^{1}\right]_{7}$ & & $1_{\mathrm{II}}^{+2}\left[2^{1}\right]_{1}$ & $1_{\mathrm{II}}^{+2}\left[2^{-1}\right]_{5}$ & & \\
\hline
\end{tabular}

$2_{Q}=1_{I, 7}^{+5}$. Using the decomposition $G=G_{1} \oplus 2(Q \ominus J(A))$ and the minimal decomposable embeddings from (36), we compose the following auxiliary table:

\begin{tabular}{|c|c|c|c|c|c|c|}
\hline $2_{G_{1}}$ & $1_{I, 0}^{+2}$ & $1_{\mathrm{I}, 4}^{-2}$ & $1_{\mathrm{I}, 2}^{+2}$ & $1_{\mathrm{I}, 6}^{-2}$ & $1_{\mathrm{I}, 6}^{+2}$ & $1_{\mathrm{I}, 2}^{-2}$ \\
\hline $2_{J(A)}$ & $1_{\mathrm{II}}^{-4}, 1_{\mathrm{I}, 0}^{-4}$ & $1_{\mathrm{II}}^{+4}, 1_{\mathrm{I}, 0}^{+4}$ & $1_{\mathrm{I}, 2}^{-4}$ & $1_{\mathrm{I}, 2}^{+4}$ & $1_{\mathrm{I}, 6}^{-4}$ & $1_{\mathrm{I}, 6}^{+4}$ \\
\hline $2_{G_{2}}, G_{2}=Q \ominus J(A)$ & & $1_{\mathrm{I}, 7}^{+1}$ & $1_{\mathrm{I}, 5}^{-1}$ & & & $1_{\mathrm{I}, 1}^{+1}$ \\
\hline $2_{G}, G=G_{1} \oplus 2 G_{2}$ & & $1_{\mathrm{I}, 4}^{-2} 2_{\mathrm{I}, 7}^{+1}$ & $1_{\mathrm{I}, 2}^{+2} 2_{\mathrm{I}, 5}^{-1}$ & & & $1_{\mathrm{I}, 2}^{-2} 2_{\mathrm{I}, 1}^{+1}$ \\
\hline $2_{G}^{\text {can }}$ & & {$\left[1^{2} 2^{-1}\right]_{7}$} & {$\left[1^{2} 2^{-1}\right]_{7}$} & & & {$\left[1^{2} 2^{-1}\right]_{7}$} \\
\hline
\end{tabular}

We see that the 2-symbols of the resulting forms $G=G_{1} \oplus 2 G_{2}$ take three distinct values, but, upon reduction to the canonical form, only the symbol $\left[1^{2} 2^{-1}\right]_{7}$ is left. In Table 6 , this symbol is placed at the intersection of the row $2_{I, 4}^{-2}$ and the column $1_{I, 7}^{+5}$. The first column of Table 6 contains the 2-symbols of the binary forms $A=2 A_{2}$, and the first row contains the 2 -symbols of forms $Q$ of dimension 5 with odd determinant. At the intersections of rows and columns, the canonical 2-symbols of the form $G$ of dimension 3 are given. An empty box means the impossibility of the respective embedding over $\mathbb{Z}_{2}$.

Tables 7 and 8 contain the embeddings of two-dimensional forms $A=q A_{q}$ (with odd and even blocks $\left.A_{q}\right), q=2^{\alpha}, \alpha \geq 2$, in $Q$ with odd determinant and $\operatorname{dim} Q=5$; they are organized similarly. Here we have used tables and formulas of Subsections 4.3 and 4.4 and also formulas (54) for subtraction of 2-symbols.

TABLE 7

\begin{tabular}{|c|c|c|c|c|c|c|}
\hline $2_{G}$ & $1_{\mathrm{I}, 7}^{+5}$ & $1_{\mathrm{I}, 3}^{-5}$ & $1_{\mathrm{I}, 5}^{-5}$ & $1_{\mathrm{I}, 1}^{+5}$ & $1_{\mathrm{I}, \pm 1}^{-5}$ & $1_{\mathrm{I}, \pm 3}^{+5}$ \\
\hline $4_{\mathrm{I}, 0}^{+2}$ & {$\left[1^{2}\right]_{0}\left[4^{1}\right]_{7}$} & {$\left[1^{2}\right]_{0}\left[4^{-1}\right]_{3}$} & {$\left[1^{2}\right]_{0}\left[4^{-1}\right]_{5}$} & {$\left[1^{2}\right]_{0}\left[4^{1}\right]_{1}$} & & \\
\hline $4_{\mathrm{I}, 4}^{-2}$ & {$\left[1^{2}\right]_{0}\left[4^{-1}\right]_{3}$} & {$\left[1^{2}\right]_{0}\left[4^{1}\right]_{7}$} & {$\left[1^{2}\right]_{0}\left[4^{1}\right]_{1}$} & {$\left[1^{2}\right]_{0}\left[4^{-1}\right]_{5}$} & & \\
\hline $4_{\mathrm{I}, 2}^{+2}$ & {$\left[1^{2}\right]_{6}\left[4^{1}\right]_{7}$} & {$\left[1^{2}\right]_{6}\left[4^{-1}\right]_{3}$} & {$\left[1^{2}\right]_{6}\left[4^{-1}\right]_{5}$} & {$\left[1^{2}\right]_{6}\left[4^{1}\right]_{1}$} & & \\
\hline $4_{\mathrm{I}, 6}^{-2}$ & {$\left[1^{2}\right]_{6}\left[4^{-1}\right]_{3}$} & {$\left[1^{2}\right]_{6}\left[4^{1}\right]_{7}$} & {$\left[1^{2}\right]_{6}\left[4^{1}\right]_{1}$} & {$\left[1^{2}\right]_{6}\left[4^{-1}\right]_{5}$} & & \\
\hline $4_{\mathrm{I}, 6}^{+2}$ & {$\left[1^{2}\right]_{2}\left[4^{1}\right]_{7}$} & {$\left[1^{2}\right]_{2}\left[4^{-1}\right]_{3}$} & {$\left[1^{2}\right]_{2}\left[4^{-1}\right]_{5}$} & {$\left[1^{2}\right]_{2}\left[4^{1}\right]_{1}$} & & \\
\hline $4_{\mathrm{I}, 2}^{-2}$ & {$\left[1^{2}\right]_{2}\left[4^{-1}\right]_{3}$} & {$\left[1^{2}\right]_{2}\left[4^{1}\right]_{7}$} & {$\left[1^{2}\right]_{2}\left[4^{1}\right]_{1}$} & {$\left[1^{2}\right]_{2}\left[4^{-1}\right]_{5}$} & & \\
\hline $4_{\mathrm{II}}^{+2}$ & $1_{\mathrm{II}}^{+2}:\left[4^{+1}\right]_{7}$ & $1_{\mathrm{II}}^{+2}:\left[4^{-1}\right]_{3}$ & $1_{\mathrm{II}}^{+2}:\left[4^{-1}\right]_{5}$ & $1_{\mathrm{II}}^{+2}:\left[4^{+1}\right]_{1}$ & & \\
\hline $4_{\mathrm{II}}^{-2}$ & $1_{\mathrm{II}}^{-2}:\left[4^{+1}\right]_{7}$ & $1_{\mathrm{II}}^{-2}:\left[4^{-1}\right]_{3}$ & $1_{\mathrm{II}}^{-2}:\left[4^{-1}\right]_{5}$ & $1_{\mathrm{II}}^{-2}:\left[4^{+1}\right]_{1}$ & & \\
\hline
\end{tabular}


TABLE 8

\begin{tabular}{|c|c|c|c|c|c|c|}
\hline $2_{G}$ & $1_{\mathrm{I}, 7}^{+5}$ & $1_{\mathrm{I}, 3}^{-5}$ & $1_{\mathrm{I}, 5}^{-5}$ & $1_{\mathrm{I}, 1}^{+5}$ & $1_{\mathrm{I}, \pm 1}^{-5}$ & $1_{\mathrm{I}, \pm 3}^{+5}$ \\
\hline$q_{\mathrm{I}, 0}^{+2}$ & {$\left[1^{2}\right]_{0}:\left[q^{1}\right]_{7}$} & {$\left[1^{2}\right]_{0}:\left[q^{-1}\right]_{3}$} & {$\left[1^{2}\right]_{0}:\left[q^{-1}\right]_{5}$} & {$\left[1^{2}\right]_{0}:\left[q^{1}\right]_{1}$} & & \\
\hline$q_{\mathrm{I}, 4}^{-2}$ & {$\left[1^{-2}\right]_{4}:\left[q^{1}\right]_{7}$} & {$\left[1^{-2}\right]_{4}:\left[q^{-1}\right]_{3}$} & {$\left[1^{-2}\right]_{4}:\left[q^{-1}\right]_{5}$} & {$\left[1^{-2}\right]_{4}:\left[q^{1}\right]_{1}$} & & \\
\hline$q_{\mathrm{I}, 2}^{+2}$ & {$\left[1^{2}\right]_{6}:\left[q^{1}\right]_{7}$} & {$\left[1^{2}\right]_{6}:\left[q^{-1}\right]_{3}$} & {$\left[1^{2}\right]_{6}:\left[q^{-1}\right]_{5}$} & {$\left[1^{2}\right]_{6}:\left[q^{1}\right]_{1}$} & & \\
\hline$q_{\mathrm{I}, 6}^{-2}$ & {$\left[1^{-2}\right]_{2}:\left[q^{1}\right]_{7}$} & {$\left[1^{-2}\right]_{2}:\left[q^{-1}\right]_{3}$} & {$\left[1^{-2}\right]_{2}:\left[q^{-1}\right]_{5}$} & {$\left[1^{-2}\right]_{2}:\left[q^{1}\right]_{1}$} & & \\
\hline$q_{\mathrm{I}, 6}^{+2}$ & {$\left[1^{2}\right]_{2}:\left[q^{1}\right]_{7}$} & {$\left[1^{2}\right]_{2}:\left[q^{-1}\right]_{3}$} & {$\left[1^{2}\right]_{2}:\left[q^{-1}\right]_{5}$} & {$\left[1^{2}\right]_{2}:\left[q^{1}\right]_{1}$} & & \\
\hline$q_{\mathrm{I}, 2}^{-2}$ & {$\left[1^{-2}\right]_{6}:\left[q^{1}\right]_{7}$} & {$\left[1^{-2}\right]_{6}:\left[q^{-1}\right]_{3}$} & {$\left[1^{-2}\right]_{6}:\left[q^{-1}\right]_{5}$} & {$\left[1^{-2}\right]_{6}:\left[q^{1}\right]_{1}$} & & \\
\hline$q_{\mathrm{II}}^{+2}$ & $1_{\mathrm{II}}^{+2}:\left[q^{+1}\right]_{7}$ & $1_{\mathrm{II}}^{+2}:\left[q^{-1}\right]_{3}$ & $1_{\mathrm{II}}^{+2}:\left[q^{-1}\right]_{5}$ & $1_{\mathrm{II}}^{+2}:\left[q^{+1}\right]_{1}$ & & \\
\hline$q_{\mathrm{II}}^{-2}$ & $1_{\mathrm{II}}^{-2}:\left[q^{+1}\right]_{7}$ & $1_{\mathrm{II}}^{-2}:\left[q^{-1}\right]_{3}$ & $1_{\mathrm{II}}^{-2}:\left[q^{-1}\right]_{5}$ & $1_{\mathrm{II}}^{-2}:\left[q^{+1}\right]_{1}$ & & \\
\hline
\end{tabular}

Using the techniques of minimal embeddings over $\mathbb{Z}_{2}$, in the same way we deduce the fact that, among the odd forms $Q=Q_{\mathrm{I}}$ of dimension 6 with odd determinant, the forms with the 2 -symbol $1_{\mathrm{I}, 0}^{-6}$ represent no binary forms $A$ with the 2 -symbol $2_{\text {II }}^{-2}$, and the forms with the 2 -symbol $1_{\mathrm{I}, 4}^{+6}$ represent no binary forms with the 2 -symbols $4_{\mathrm{II}}^{ \pm 2}, q_{\mathrm{I}, 0}^{+2}$, $q_{\mathrm{I}, 4}^{-2}, q_{\mathrm{I}, 6}^{ \pm 2} q_{\mathrm{I}, 2}^{ \pm 2}, q_{\mathrm{II}}^{ \pm 2}$ for $q=2^{\alpha}, \alpha \geq 3$. The other forms $Q=Q_{\mathrm{I}}$ with odd determinant and dimension at least 6 represent the forms $A=q A_{q}, q \geq 2, \operatorname{dim} A_{q}=2$ over $\mathbb{Z}_{2}$.

Next, consider the representations of the forms $A=A_{1}$, $\operatorname{dim} A=2$, by odd forms $Q=Q_{\mathrm{I}}$ with odd determinant. The calculation of the forms $G=Q \ominus A_{1}$, see (27), reduces to the subtraction formulas (54,1) and (54,3) for 2-symbols, where $A=A_{1}$ ranges over all forms in (35) and (45). For $\operatorname{dim} Q \geq 5$, the resulting 2 -symbols of $G$ always exist. Consequently, the binary forms $A=A_{1}$ are primitively embedded in the odd forms $Q=Q_{1}, \operatorname{dim} Q \geq 5$, over $\mathbb{Z}_{2}$.

Using the minimal embeddings of the one-dimensional forms $A=q A_{q}$ as in Subsection 4.2 and formula (34), we consider representations of the forms $A=A_{1} \oplus q A_{q}$, $\operatorname{dim} A_{1}=\operatorname{dim} A_{q}=1, q=2^{\alpha}, \alpha \geq 1$, by odd forms $Q=Q_{\mathrm{I}}, \operatorname{dim} Q \geq 5$, with odd determinant. Calculations show that the forms $A=A_{1} \oplus q A_{q}$ for $q=2,4$ are primitively embedded in odd forms $Q=Q_{\mathrm{I}}, \operatorname{dim} Q \geq 5$, with odd determinant. If $q \geq 8$, then the forms $A$ with the 2 -symbols $\left[1^{1}\right]_{1}:[q]_{\mathrm{I}, t}^{ \pm 1},\left[1^{1}\right]_{7}:[q]_{\mathrm{I}, t}^{ \pm 1},\left[1^{-1}\right]_{3}:[q]_{\mathrm{I}, t}^{ \pm 1}$, and $\left[1^{-1}\right]_{5}:[q]_{\mathrm{I}, t}^{ \pm 1}$ are not primitively represented by forms $Q$ with the 2 -symbols $1_{\mathrm{I}, 5}^{+5}, 1_{\mathrm{I}, 3}^{+5}, 1_{\mathrm{I}, 7}^{-5}, 1_{\mathrm{I}, 1}^{-5}$, respectively; if $q \geq 8$ and $\operatorname{dim} Q \geq 6$, then primitive representations of the forms $A=A_{1} \oplus q A_{q}$ exist over $\mathbb{Z}_{2}$.

It remains to consider the representations of the forms $A=q_{1} A_{q_{1}} \oplus q_{2} A_{q_{2}}, \operatorname{dim} A_{q_{1}}=$ $\operatorname{dim} A_{q_{2}}=1, q_{2}>q_{1} \geq 2, q_{i}=2^{\alpha_{i}}, i=1,2$, by forms $Q=Q_{\mathrm{I}}$, $\operatorname{dim} Q \geq 5$, with odd determinant (see Subsection 4.5). The forms with the 2 -symbols $1_{I, 5}^{+5}, 1_{I, 1}^{-5}, 1_{I, 7}^{-5}$, $1_{I, 3}^{+5}$ do not primitively represent the binary forms $A=q_{1} A_{q_{1}} \oplus q_{2} A_{q_{2}}, q_{2}>q_{1} \geq 4$, over $\mathbb{Z}_{2}$. The next forms with the 2 -symbol $1_{I, 4}^{+6}$ do not primitively represent the forms $A=q_{1} A_{q_{1}} \oplus q_{2} A_{q_{2}}, q_{2}>q_{1} \geq 8$. The other forms $Q=Q_{\mathrm{I}}, \operatorname{dim} Q \geq 5$, with odd determinant represent the forms $A=q_{1} A_{q_{1}} \oplus q_{2} A_{q_{2}}, q_{2}>q_{1} \geq 2$, over $\mathbb{Z}_{2}$.

If $n=\operatorname{dim} Q$ is odd and $Q=Q_{1}$, then the type of the form $Q$ may only be odd, i.e., $Q=Q_{\mathrm{I}}$. For even dimensions $n$, also even forms $Q=Q_{\mathrm{II}}$ arise, which represent only even forms $A=A_{\mathrm{II}}$ over $\mathbb{Z}_{2}$. Consequently, the even forms $Q=Q_{\mathrm{II}}$ are not primitively $2 \sim$ universal. 
Proposition 3. Let $Q$ be a positive definite, classically integral quadratic form of dimension at least 5 with odd and square-free determinant. If the 2-symbol of the form $Q$ satisfies the $2 \sim$ universality condition in Theorem 5 and the p universality condition in Theorem 4 for every odd prime $p$ that divides $|Q|$, then $Q$ is a locally primitively 2-universal form.

Proof. We must show that all forms $Q$ as in Proposition 3 are primitively $p \sim$ universal for all $p=-1,2,3,5, \ldots$ Over $\mathbb{Z}_{-1}$, the positive definite forms $Q$ primitively represent the positive definite forms $A$. (In the present context, $\mathbb{R}$ is denoted by $\mathbb{Z}_{-1}$.)

For $p=2$, if $p|| Q \mid$ where $|Q|$ is odd and square-free, the conditions of $p \sim$ universality are described in Theorems 5 and 4 , respectively.

It remains to consider the case where $p$ is odd and $p \nmid|Q|$. In accordance with the behavior of the binary forms $A$ over $\mathbb{Z}_{p}$, we obtain three kinds of minimal embeddings $J(A)$ :

$$
\begin{aligned}
& A=A_{1}=A_{11} \oplus A_{12} \hookrightarrow J(A)=A_{11} \oplus A_{12}, \\
& A=A_{1} \oplus p^{\alpha_{1}} A_{p^{\alpha_{1}}} \hookrightarrow J(A)=A_{1} \oplus J\left(p^{\alpha_{1}} A_{p^{\alpha_{1}}}\right), \quad \alpha_{1} \geq 1, \\
& A=p^{\alpha_{2}} A_{p^{\alpha_{2}}} \oplus p^{\alpha_{3}} A_{p^{\alpha_{3}}} \hookrightarrow J(A)=J\left(p^{\alpha_{2}} A_{p^{\alpha_{2}}}\right) \oplus J\left(p^{\alpha_{3}} A_{p^{\alpha_{3}}}\right), \quad \alpha_{3} \geq \alpha_{2} \geq 1,
\end{aligned}
$$

of dimensions 2, 3 and 4, respectively (see Subsection 3.1). Since we consider forms $Q$ with $\operatorname{dim} Q \geq 5$, the differences $Q \ominus J(A)$ always exist (see [23]). Consequently, the binary forms $A$ are primitively embedded in $Q=Q_{1}, \operatorname{dim} Q \geq 5$, over an odd $\operatorname{ring} \mathbb{Z}_{p}$, $p \nmid|Q|$.

5.2. Next we prove Theorem 2 , stated in the Introduction.

Proof of Theorem 2. If $h(Q)=1$, then the form $Q$ satisfies the following global principle over $\mathbb{Z}$ : a form $A$ is primitively represented by $Q$ over $\mathbb{Z}$ if and only if $A$ is primitively represented by $Q$ over every ring $\mathbb{Z}_{p}, p=-1,2,3,5, \ldots$. Consequently, a one-class form is primitively 2-universal if and only if it is locally primitively 2-universal. If $h(Q)>1$, then the local 2-universality of forms $Q$ enables us to talk of the 2-universal genus $[Q]$.

First, we consider primitive representations of forms over the $\operatorname{ring} \mathbb{Z}_{2}$. If $Q=Q_{\mathrm{II}}$ is an even form, then the form $A$ to be represented is also even. Consequently, if we adjoin any odd binary form of odd determinant, for example, $\left(\begin{array}{ll}1 & 0 \\ 0 & 1\end{array}\right)$, to the critical forms, then the even forms $Q=Q_{\mathrm{II}}$ will be removed from our consideration.

Analyzing Tables 6-8 and the behavior of the forms $Q=Q_{\mathrm{I}}$ of dimension 6 and odd determinant $|Q|$, we conclude that for primitive representability over $\mathbb{Z}_{2}$ it is necessary to add the forms $2\left(\begin{array}{ll}2 & 1 \\ 1 & 2\end{array}\right)$ and $8\left(\begin{array}{ll}1 & 0 \\ 0 & 7\end{array}\right)$ to the critical forms. The representability of the first form removes the forms with the 2 -symbols $1_{\mathrm{I}, \pm 3}^{+5}, 1_{\mathrm{I}, \pm 1}^{-5}$, and $1_{\mathrm{I}, 0}^{-6}$ from our consideration, and the representability of the second removes the forms with the 2 -symbol $1_{\mathrm{I}, 4}^{+6}$.

Next, we consider primitive representability over $\mathbb{Z}_{p}$, where $p$ is an odd prime that divides the square-free determinant $|Q|$. In accordance with Table 1 , to the critical forms we must add any form of type 3 , for example, $p^{2}\left(\begin{array}{ll}1 & 0 \\ 0 & 1\end{array}\right)$; this condition removes the forms $Q, \operatorname{dim} Q=5$, with $\epsilon_{1}(Q)=-1$.

If $p=-1$ or $p$ is an odd prime not dividing $|Q|$, then primitive representations of binary forms by forms $Q, \operatorname{dim} Q \geq 5$, always exist over $\mathbb{Z}_{p}$ (see the proof of Proposition 3 ).

Remark 1. It should be noted that the choice of critical forms in Theorem 2 is nonunique.

5.3. B. M. Kim, M.-H. Kim, and B.-K. Oh have found all 2-universal, classically integral, quadratic forms $Q$ of dimension 5; they are given in Table 9 together with their local invariants. All forms $Q$ in Table 9 , except for the form $Q_{6}^{2}$, are one-class forms. Using Theorems 4 and 5 , we obtain the following result. 


\section{TABLE 9}

$$
\begin{array}{lll}
Q_{1}: x_{1}^{2}+x_{2}^{2}+x_{3}^{2}+x_{4}^{2}+x_{5}^{2}, & 2_{Q_{1}}=1_{\mathrm{I}, 5}^{+5}, \\
Q_{2}^{1}: x_{1}^{2}+x_{2}^{2}+x_{3}^{2}+x_{4}^{2}+2 x_{5}^{2}, & 2_{Q_{2}^{1}}=1_{\mathrm{I}, 4}^{+4} 2_{\mathrm{I}, 1}^{+1}, & \\
Q_{3}^{1}: x_{1}^{2}+x_{2}^{2}+x_{3}^{2}+x_{4}^{2}+3 x_{5}^{2}, & 2_{Q_{3}^{1}}=1_{\mathrm{I}, 7}^{-5}, & 3_{Q_{3}^{1}}=1^{+4} 3^{+1}, \\
Q_{4}: x_{1}^{2}+x_{2}^{2}+x_{3}^{2}+2 x_{4}^{2}+2 x_{5}^{2}, & 2_{Q_{4}}=1_{\mathrm{I}, 3}^{+3} 2_{\mathrm{I}, 2}^{+2}, & \\
Q_{6}^{1}: x_{1}^{2}+x_{2}^{2}+x_{3}^{2}+2 x_{4}^{2}+3 x_{5}^{2}, & 2_{Q_{6}^{1}}=1_{\mathrm{I}, 6}^{-4} 2_{\mathrm{I}, 1}^{+1}, & 3_{Q_{6}^{1}}=1^{-4} 3^{+1}, \\
Q_{2}^{2}: x_{1}^{2}+x_{2}^{2}+2 x_{3}^{2}+2 x_{4}^{2}+2 x_{5}^{2}+2 x_{3} x_{4}+2 x_{4} x_{5}, & 2_{Q_{2}^{2}}=1_{\mathrm{I}, 2}^{-4} 2_{\mathrm{I}, 3}^{-1}, & \\
Q_{3}^{2}: x_{1}^{2}+x_{2}^{2}+x_{3}^{2}+2 x_{4}^{2}+2 x_{5}^{2}+2 x_{4} x_{5}, & 2_{Q_{3}^{2}}=1_{\mathrm{I}, 3}^{-5}, & \\
Q_{5}: x_{1}^{2}+x_{2}^{2}+x_{3}^{2}+2 x_{4}^{2}+3 x_{5}^{2}+2 x_{4} x_{5}, & 2_{Q_{5}}=1_{\mathrm{I}, 5}^{-5}, & 5_{Q_{5}}=1^{-4} 3^{-1}, \\
Q_{6}^{2}: x_{1}^{2}+x_{2}^{2}+2 x_{3}^{2}+2 x_{4}^{2}+2 x_{5}^{2}+2 x_{4} x_{5}, & 2_{Q_{6}^{2}}=1_{\mathrm{I}, 2}^{-4} 2_{\mathrm{I}, 1}^{+1}, & 3_{Q_{6}^{2}}=1^{+4} 3^{-1}, \\
Q_{7}: x_{1}^{2}+x_{2}^{2}+2 x_{3}^{2}+2 x_{4}^{2}+3 x_{5}^{2}+2 x_{3} x_{4}+2 x_{4} x_{5}, & 2_{Q_{7}}=1_{\mathrm{I}, 7}^{+5}, & 7_{Q_{7}}=1^{-4} 7^{-1}, \\
Q_{9}: x_{1}^{2}+x_{2}^{2}+3 x_{3}^{2}+2 x_{4}^{2}+2 x_{5}^{2}+2 x_{4} x_{5}, & 2_{Q_{9}}=1_{\mathrm{I}, 5}^{+5}, & 3_{Q_{9}}=1^{-3} 3^{-2} .
\end{array}
$$

Theorem 6. The forms $Q_{3}^{2}, Q_{5}, Q_{6}^{1}, Q_{7}$ are not primitively 2-universal, because they are not primitively $p \sim$ universal, $p \neq 2$. The forms $Q_{1}, Q_{3}^{1}$, and $Q_{9}$ are not primitively 2-universal, because they are not primitively $2 \sim$ universal.

For the remaining four forms $Q_{2}^{1}, Q_{2}^{2}, Q_{4}, Q_{6}^{2}$ in Table 9 , which are primitively $p \sim$ universal for every odd $p$, the problem reduces to primitive representations over $\mathbb{Z}_{2}$, which calls for further investigation.

\section{REFERENCES}

[1] M. Bhargava, On the Conway-Schneeberger fifteen theorem, Quadratic Forms and their Applications (E. Bayer-Fluckiger, D. Lewis, A. Ranicki, eds.) (Dublin, 1999), Contemp. Math., vol. 272, Amer. Math. Soc., Providence, RI, 2000, pp. 27-37. MR1803359 (2001m:11050)

[2] B.-K. Oh, Universal $\mathbb{Z}$-lattices of minimal rank, Proc. Amer. Math. Soc. 128 (2000), no. 3, $683-689$. MR.1654105 (2000e:11041)

[3] B. M. Kim, M.-H. Kim, and B.-K. Oh, 2-universal positive definite integral quinary quadratic forms, Integral Quadratic Forms and Lattices (Seoul, 1998), Contemp. Math., vol. 249, Amer. Math. Soc., Providence, RI, 1999, pp. 51-62. MR.1732349 (2001c:11047)

[4] W. K. Chan, M.-H. Kim, and S. Raghavan, Ternary universal integral quadratic forms over real quadratic fields, Japan. J. Math. 22 (1996), 263-273. MR1432376 (97m:11051)

[5] A. Earnest and A. Khosravani, Universal positive quaternary quadratic lattices over totally real number fields, Mathematika 44 (1997), no. 2, 342-347. MR1600557 (99a:11045)

[6] B. M. Kim, Finiteness of real quadratic fields which admit positive integral diagonal septanary universal forms, Manuscripta Math. 99 (1999), no. 2, 181-184. MR1697212 (2000h:11032)

[7] _ Universal octonary diagonal forms over some real quadratic fields, Comment. Math. Helv. 75 (2000), no. 3, 410-414. MR.1793795 (2001m:11046)

[8] H. Maass, Über die Darstellung total positiver Zahlen des Körpers $R(\sqrt{5})$ als Summe von drei Quadraten, Abh. Math. Sem. Hansischen Univ. 14 (1941), 185-191. MR0005505 (3:163a)

[9] B.-K. Oh, The representations of quadratic forms by almost universal forms of higher rank, Math. Z. 244 (2003), 399-413. MR.1992544 (2004m:11053)

[10] H. D. Kloosterman, On the representation of numbers in the form $a x^{2}+b y^{2}+c z^{2}+d t^{2}$, Acta Math. 49 (1926), 407-464.

[11] G. Pall, The completion of a problem of Kloosterman, Amer. J. Math. 68 (1946), 47-58. MR0014377 $(7: 275 \mathrm{a})$

[12] G. Pall and A. Ross, An extension of a problem of Kloosterman, Amer. J. Math. 68 (1946), 59-65. $\operatorname{MR} 0014378(7: 275 b)$ 
[13] J. Bochnak and B.-K. Oh, Almost-universal quadratic forms: an effective solution of a problem of Ramanujan, Duke Math. J. 147 (2009), no. 1, 131-156. MR.2494458 (2009m:11050)

[14] M. Bhargava, Finiteness theorems for quadratic forms, Preprint.

[15] B. M. Kim, M.-H. Kim, and B.-K. Oh, A finiteness theorem for representability of quadratic forms by forms, J. Reine Angew. Math. 581 (2005), 23-30. MR2132670 (2006a:11044)

[16] B.-K. Oh, Positive definite n-regular quadratic forms, Invent. Math. 170 (2007), 421-453. MR.2342641 (2009b:11065)

[17] A. G. Earnest, The representation of binary quadratic forms by positive definite quaternary quadratic forms, Trans. Amer. Math. Soc. 345 (1994), no. 2, 853-863. MR1264145 (95a:11034)

[18] N. Budarina, On primitively universal quadratic forms, Lith. Math. J. 50 (2010), no. 2, $140-163$. MR2653643 (2011d:11086)

[19] V. G. Zhuravlev, Representation of a form by the genus of quadratic forms, Algebra i Analiz 8 (1996), no. 1, 21-112; English transl., St. Petersburg Math. J. 8 (1997), no. 1, 15-84. MR.1392012 (97d:11059)

[20] - Orbits of representations of numbers by local quadratic forms, Trudy Mat. Inst. Steklova 218 (1997), 151-164; English transl., Proc. Steklov Inst. Math. 1997, no. 3 (218), 146-159. MR.1642373 (2001f:11055)

[21] _ Embedding p-elementary lattices, Izv. Ross. Akad. Nauk Ser. Mat. 63 (1999), no. 1, 77-106; English transl., Izv. Math. 63 (1999), no. 1, 73-102. MR.1701839 (2000h:11036)

[22] _ Primitive embeddings in local lattices with prime determinant, Algebra i Analiz 11 (1999), no. 1, 87-117; English transl., St. Petersburg Math. J. 11 (2000), no. 1, 67-90. MR1691081 (2000f:11084)

[23] J. H. Conway and N. J. A. Sloane, Sphere packings, lattices and groups, 3rd ed., Grundlehren Math. Wiss., Bd. 290, Springer-Verlag, New York, 1999. MR1662447 (2000b:11077)

[24] J. W. S. Cassels, Rational quadratic forms, London Math. Soc. Monogr., vol. 13, Acad. Press, Inc., London-New York, 1978. MR0522835 (80m:10019)

[25] V. G. Zhuravlev, Deformation of quadratic Diophantine systems, Izv. Ross. Akad. Nauk Ser. Mat. 65 (2001), no. 6, 15-56; English transl., Izv. Math. 65 (2001), no. 6, 1085-1126. MR1892903 (2004a:11028)

Khabarovsk Division, Institute of Applied Mathematics, Russian Academy of Sciences, 54 Dzerzhinsky Street, Khabarovsk 680000, Russia

E-mail address: buda77@mail.ru

Received 5/FEB/2010

Translated by N. B. LEBEDINSKAYA 